Article

\title{
Quality Evaluation of Pseudostellariae Radix Based on Simultaneous Determination of Multiple Bioactive Components Combined with Grey Relational Analysis
}

\author{
Yujiao Hua, Shengnan Wang, Chuan Chai, Zixiu Liu, Xunhong Liu *, Lisi Zou, Qinan Wu, \\ Hui Zhao and Yan Ying \\ College of Pharmacy, Nanjing University of Chinese Medicine, Nanjing 210023, China; \\ 20141550@njucm.edu.cn (Y.H.); jshmwsn@163.com (S.W.); echo_0523@hotmail.com (C.C.); \\ liuzixiu3221@126.com (Z.L.); zlstcm@126.com (L.Z.); qnwyjs@163.com (Q.W.); zhaohui_199301@163.com (H.Z.); \\ yanying931y@163.com (Y.Y.) \\ * Correspondence: liuxunh1959@sohu.com; Tel./Fax: +86-25-8581-1524
}

Academic Editor: Derek J. McPhee

Received: 1 December 2016; Accepted: 21 December 2016; Published: 26 December 2016

\begin{abstract}
Pseudostellariae Radix (PR) is an important traditional Chinese herbal medicine (TCM) with vast clinical consumption because of its positive effects. However, little attention has been devoted to simultaneous analysis of its bioactive components for quality control of PR based on its different harvesting times, different growing habitats, and different processing methods. In this research, the quality of PR was evaluated based on simultaneous determination of multiple bioactive components combined with grey relational analysis (GRA). A reliable method based on ultra-fast liquid chromatography tandem triple quadrupole mass spectrometry (UFLC-QTRAP-MS/MS) was established to simultaneously determine the contents of 30 components in PR, including two cyclopeptides, 12 nucleosides, and 16 amino acids. Furthermore, grey relational analysis was performed to evaluate the quality of PR samples according to the contents of these 30 components. The results showed that the quality of PR harvested in 6 August 2013, cultivated in Jurong, Jiangsu, and treated by oven drying $60^{\circ} \mathrm{C}$ was better than that of other PR samples. The proposed method is useful for the overall assessment on the quality of PR, and this study provides valuable information for revealing the dynamic change laws of metabolite accumulation in PR and choosing the most suitable harvesting time and reasonable processing method of PR to obtain the best quality.
\end{abstract}

Keywords: Pseudostellariae Radix; UFLC-QTRAP-MS/MS; multiple bioactive components; simultaneous quantitation; GRA

\section{Introduction}

Pseudostellariae Radix (PR) is the dry tuberous root of Pseudostellaria heterophylla (Miq.) Pax ex Pax et Hoffm. [1]. It is a staple traditional Chinese herbal medicine which has the functions of strengthening the spleen, replenishing Qi, moistening lungs, and producing fluids. This medicinal herb is widely consumed clinically for its positive effects. It is reported that PR can be used for inappetence [2], thirst [3], debility [4], diabetes [5], and weakness after illness [6], and it has become an important medicine to cure loss of appetite in children due to spleen deficiency. Phytochemical studies indicate that cyclopeptides, amino acids, and nucleosides are the main bioactive compounds in PR [7-9]. Some studies have proved that these PR components have various biological activities. For example, cyclopeptides inhibit melaninogenesis and tyrosinase activity [10]; amino acids are the important medical components in PR, which have nourishing and tonifying functions [11]; nucleosides are the 
active ingredients that enhance immune activity [12]. The synergistic action of these components is considered to be responsible for the broad clinical effects of PR. As PR resources are declining and the demand for original medicinal materials is rising annually, the government has established a large-scale cultivation base for PR. However, due to differences in the ecological environment, the accumulation of active components and the quality of the PR material from different habitats show great differences. The harvesting time and primary processing of PR are the important factors which are closely related to the quality of this TCM. The different harvesting times and various processing methods can affect the chemical components in PR. On account of different harvesting times, different habitats, and different processing methods, it is difficult to achieve their standardization to ensure the effectiveness in clinical use. In view of the current situation, it is necessary to develop a rapid and reliable method to evaluate the bioactive components for quality control of PR.

In recent years, a variety of analytical methods have focused on the determination of the contents of cyclopeptides or nucleosides or amino acids in PR. High-performance liquid chromatography (HPLC) [13], gas chromatography coupled to mass spectrometry (GC/MS) [14], and high performance capillary electrophoresis (HPCE) [15] have all been reported as tools for PR quality assessment. However, the contents of only cyclopeptides or nucleosides or amino acids cannot accurately represent the quality of the complex herbal products, and a method for the simultaneous quantitative determination of cyclopeptides, nucleosides, and amino acids has not been established. Therefore, a universal method for the quantitative determination of multiple components in PR is necessary and convenient for their quality control. The ultra-fast liquid chromatography tandem triple quadrupole mass spectrometry (UFLC-QTRAP-MS/MS) method, which uses multiple reaction monitoring (MRM) for quantitative analysis, is highly sensitive and fast. MRM is a data acquisition technology with high sensitivity, accuracy, and throughput, which uses one transition each from the precursor to the reporter ions for every compound, respectively. This technology has been widely used in the analysis of complex TCMs [16-18].

In this work, an accurate and reliable method based on UFLC-QTRAP-MS/MS for the simultaneous determination of multiple bioactive components in PR was established and validated. A total of 30 compounds, including two cyclopeptides, 12 nucleosides, and 16 amino acids were selected as the marker compounds. Multiple reaction monitoring (MRM), a tandem MS scan mode unique to triple quadrupole MS instrumentation, was employed for quantification in the present study. The proposed method was successfully applied to analyze fifteen PR samples from different harvesting times, different habitats, and treated with different processing methods. Furthermore, grey relational analysis (GRA), a quantitative comparative analysis method which is wildly used in the quality assessment in TCMs $[19,20]$, was performed to evaluate the samples according to the contents of the 30 marker compounds. This method does not need too much sample size and typical distribution, and also has the advantage of needing a small amount of computation, and the results will accord with the quantitative results. To the best of our knowledge, this is the most comprehensive published report in the quantitative analysis of PR.

\section{Results and Discussion}

\subsection{Optimization of Extraction Conditions}

The extraction conditions, including extraction method (ultrasonic extraction and refluxing extraction), extraction solvent (water, 25\% methanol, 50\% methanol, 75\% methanol, and 100\% methanol), solvent to sample ratios (80:1, 100:1, 200:1, and 250:1 (v/w)), and extraction time (45 min, $60 \mathrm{~min}, 75 \mathrm{~min}$, and $90 \mathrm{~min}$ ) were optimized in order to obtain the most satisfactory extraction efficiency. The results showed that ultrasonic extraction with a 200:1 ratio of water for 60 min at room temperature was sufficient for complete extraction of the target compounds. 


\subsection{Optimization of UFLC Conditions}

The amino acids, nucleosides, and cyclopeptides in PR all have large hydrophilicity, so hydrophilic chromatography columns have a strong retention ability as well as good resolution for these compounds; thus, in order to achieve rapid and efficient analysis, an XBridge Amide $(2.1 \mathrm{~mm} \times 100 \mathrm{~mm}$, $3.5 \mu \mathrm{m}$ ) column (Waters, Milford, MA, USA) was employed for this analysis. Different mobile phases (including acetonitrile-water, methanol-water, acetonitrile-formic acid solution, methanol-formic acid solution, acetonitrile with formic acid solution-formic acid solution, and methanol with formic acid solution-formic acid solution), flow rate $(0.4,0.5$, and $0.6 \mathrm{~mL} / \mathrm{min}$ ) as well as column temperature $\left(25,30\right.$, and $\left.35{ }^{\circ} \mathrm{C}\right)$ were examined and compared. As a result, it was determined that acetonitrile with $0.2 \%$ formic acid $-0.2 \%$ formic acid solution at a flow rate of $0.6 \mathrm{~mL} / \mathrm{min}$ with the column temperature of $30{ }^{\circ} \mathrm{C}$ resulted in satisfactory separation in a short analysis time.

\subsection{Optimization of $M S$ Conditions}

In order to develop a sensitive and accurate quantitative method, all the compounds were examined separately in direct infusion mode by a full-scan MS method in both positive and negative mode, and all analytes showed maximum sensitivity when the instrument was operated in the positive ion mode. The parameters of fragmentor voltage (FV) and collision energy (CE) were optimized to achieve the most abundant, specific, and stable transition for each compound. The retention time (RT) and MS information for each analytes including $[\mathrm{M}+\mathrm{H}]^{+}$, precursor and product ions, FV and CE are shown in Table 1.

Table 1. Retention time, related MS data of the target compounds.

\begin{tabular}{|c|c|c|c|c|c|c|}
\hline Coumpounds & RT (min) & {$[\mathbf{M}+\mathbf{H}]^{+}(m / z)$} & Precursor Ion & Product Ion & FV & $\mathrm{CE}$ \\
\hline Heterophyllin A & 0.50 & 728.43 & 728.4 & 70.05 & 211 & 119 \\
\hline Heterophyllin B & 0.55 & 779.44 & 779.39 & 70.05 & 226 & 117 \\
\hline Uracil & 0.75 & 112.09 & 113.04 & 70 & 111 & 21 \\
\hline Thymidine & 0.78 & 242.23 & 243.1 & 127.07 & 61 & 13 \\
\hline Hypoxanthine & 1.11 & 136.11 & 137.05 & 137.05 & 51 & 24 \\
\hline Uridine & 1.13 & 244.2 & 244.9 & 113 & 103 & 13 \\
\hline Adenine & 1.31 & 135.13 & 136.06 & 136 & 51 & 24 \\
\hline Adenosine & 1.39 & 267.24 & 268.1 & 136.07 & 86 & 23 \\
\hline Phenylalanine & 1.4 & 165.19 & 166.1 & 120.05 & 56 & 14 \\
\hline Inosine & 1.59 & 268.23 & 269 & 137.07 & 46 & 15 \\
\hline Valine & 1.66 & 117.15 & 118.09 & 72.06 & 54 & 10 \\
\hline Methionine & 1.72 & 149.21 & 150.06 & 104.03 & 91 & 10 \\
\hline Dideoxyguanosine & 1.79 & 267.2 & 268.1 & 152.1 & 61 & 15 \\
\hline Leucine & 1.81 & 131.18 & 132.1 & 86.05 & 98 & 10 \\
\hline Tyrosine & 1.85 & 182.1 & 182.16 & 136.08 & 46 & 17 \\
\hline Dideoxycytidine & 1.87 & 227.3 & 228.2 & 112.05 & 76 & 13 \\
\hline Isoleucine & 1.92 & 131.18 & 132.1 & 86.05 & 64 & 10 \\
\hline Guanine & 1.95 & 151.12 & 152 & 135 & 51 & 25 \\
\hline Proline & 2.31 & 115.13 & 116.07 & 70.02 & 68 & 10 \\
\hline Guanosine & 2.45 & 283.24 & 284.3 & 152 & 62 & 15 \\
\hline Alanine & 2.66 & 89.09 & 90.06 & 44.02 & 79 & 10 \\
\hline Cytidine & 2.93 & 243.22 & 244.09 & 112 & 61 & 10 \\
\hline Glycine & 3.45 & 75.07 & 76.04 & 30 & 73 & 6 \\
\hline Threonine & 3.98 & 119.12 & 120.07 & 74 & 93 & 20 \\
\hline Aspartic acid & 4.25 & 133.1 & 134.05 & 87.96 & 59 & 10 \\
\hline Serine & 4.42 & 105.09 & 106.05 & 59.99 & 67 & 8 \\
\hline Arginine & 5.07 & 174.2 & 175.12 & 70.02 & 88 & 18 \\
\hline Histidine & 5.11 & 155 & 156.08 & 110.03 & 95 & 16 \\
\hline Lysine & 5.12 & 146.19 & 147.11 & 83.91 & 66 & 14 \\
\hline Glutamic acid & 5.14 & 147.13 & 147.08 & 83.92 & 83 & 14 \\
\hline
\end{tabular}

\subsection{UFLC Method Validation}

All method validations for quantification were performed using the UFLC-QTRAP-MS/MS technique. The data of each method validation are summarized in Table 2 . The calibration curves exhibited good linearity $\left(r^{2}>0.9947\right)$ within the test range. 
Table 2. Regression equation, LOD and LOQ, precision, repeatability, stability and recovery of 30 investigated compounds.

\begin{tabular}{|c|c|c|c|c|c|c|c|c|c|c|c|c|c|c|c|c|}
\hline \multirow{3}{*}{ No. } & \multirow{3}{*}{ Compounds } & \multirow{3}{*}{ Regression Equation } & \multirow{3}{*}{$r^{2}$} & \multirow{3}{*}{$\begin{array}{c}\text { Liner Range } \\
\text { (ng/mL) }\end{array}$} & \multirow{3}{*}{$\begin{array}{c}\mathrm{LOQ} \\
\text { (ng/mL) }\end{array}$} & \multirow{3}{*}{$\begin{array}{l}\text { LOD } \\
\text { (ng/mL) }\end{array}$} & \multicolumn{2}{|c|}{ Precision RSD (\%) } & \multirow{3}{*}{$\begin{array}{c}\text { Repeatability } \\
\text { RSD }(\%)(n=6)\end{array}$} & \multirow{3}{*}{$\begin{array}{l}\text { Stability } \\
\text { RSD (\%) }\end{array}$} & \multicolumn{6}{|c|}{ Recovery $(\%)(n=3)$} \\
\hline & & & & & & & \multirow{2}{*}{$\begin{array}{c}\text { Intar-Day } \\
(n=6)\end{array}$} & \multirow{2}{*}{$\begin{array}{c}\text { Inter-Day } \\
(n=6)\end{array}$} & & & \multicolumn{2}{|c|}{ Low } & \multicolumn{2}{|c|}{ Medium } & \multicolumn{2}{|c|}{ High } \\
\hline & & & & & & & & & & & Mean & RSD & Mean & RSD & Mean & RSD \\
\hline 1 & Glycine & $Y=138 X+4880$ & 0.9991 & $20.04-2004$ & 10.02 & 4.01 & 2.09 & 3.47 & 2.66 & 3.70 & 98.75 & 1.4 & 99.7 & 1.81 & 100.79 & 1.19 \\
\hline 2 & Alanine & $\mathrm{Y}=1860 \mathrm{X}+222000$ & 0.9999 & $186.84-18684$ & 93.40 & 31.13 & 2.49 & 2.59 & 1.45 & 1.02 & 100.78 & 0.92 & 102 & 1.11 & 101.32 & 1.2 \\
\hline 3 & Serine & $\mathrm{Y}=872 \mathrm{X}+52100$ & 0.9996 & $4.93-4930$ & 4.93 & 1.64 & 2.10 & 3.48 & 3.31 & 2.67 & 97.89 & 1.72 & 102.76 & 2.55 & 101.3 & 1.52 \\
\hline 4 & Proline & $Y=10100 X-459000$ & 0.9995 & $30.06-6012$ & 15.03 & 7.52 & 1.82 & 2.54 & 1.38 & 3.67 & 103.53 & 1.06 & 102.22 & 2.72 & 99.34 & 2.3 \\
\hline 5 & Valine & $\mathrm{Y}=10100 \mathrm{X}+136000$ & 0.9992 & $10.06-2012$ & 0.40 & 0.13 & 2.96 & 3.07 & 2.12 & 3.20 & 99.05 & 2.24 & 101.64 & 2.35 & 102.28 & 1.84 \\
\hline 6 & Threonine & $Y=1250 X+70400$ & 0.9991 & $81.28-4064$ & 40.64 & 20.32 & 2.01 & 2.61 & 3.14 & 1.33 & 101.68 & 1.79 & 101.08 & 1.76 & 102.58 & 2.39 \\
\hline 7 & Leucine & $\mathrm{Y}=27700 \mathrm{X}+403000$ & 0.9979 & 4.94-988 & 1.98 & 0.66 & 2.1 & 3.79 & 2.89 & 3.77 & 103.04 & 1.09 & 102.19 & 2.11 & 99.17 & 2.75 \\
\hline 8 & Isoleucine & $\mathrm{Y}=27900 \mathrm{X}+403000$ & 0.9979 & $4.91-982$ & 1.96 & 0.65 & 2.88 & 4.07 & 1.14 & 1.11 & 104.36 & 2.31 & 100.87 & 4.72 & 100.44 & 4.18 \\
\hline 9 & Aspartic acid & $Y=1120 X+36200$ & 0.9998 & $29.7-5940$ & 11.88 & 3.57 & 1.55 & 1.93 & 1.83 & 3.57 & 98.92 & 2.62 & 98.97 & 2.34 & 99.89 & 1.71 \\
\hline 10 & Glutamic acid & $Y=4740 X+99900$ & 1.0000 & $20.28-4056$ & 4.06 & 0.81 & 1.02 & 2.57 & 2.46 & 3.12 & 101.76 & 2.39 & 100.2 & 1.73 & 99.86 & 1.58 \\
\hline 11 & $\begin{array}{l}\text { Lysine } \\
\text { L }\end{array}$ & $Y=3040 X+43100$ & 0.9996 & $24.70-4940$ & 4.94 & 2.47 & 0.61 & 2.98 & 2.53 & 1.34 & 98.74 & 4.23 & 101.59 & 2.56 & 99.41 & 1.79 \\
\hline 12 & Methionine & $Y=4160 X+3600$ & 0.9998 & $0.996-99.6$ & 0.50 & 0.20 & 1.75 & 3.37 & 1.01 & 3.84 & 98.09 & 4.76 & 102.97 & 4.52 & 99.74 & 3.04 \\
\hline 13 & Histidine & $Y=12400 X+91600$ & 1.0000 & $19.76-1976$ & 9.88 & 3.95 & 1.55 & 2.43 & 2.55 & 3.58 & 97.8 & 2.31 & 100.08 & 1.52 & 100.83 & 1.47 \\
\hline 14 & Phenylalanine & $\mathrm{Y}=18800 \mathrm{X}+102000$ & 0.9999 & $4.97-994$ & 1.99 & 0.50 & 1.31 & 3.58 & 1.47 & 2.40 & 99.03 & 3.06 & 101.54 & 3.63 & 100.81 & 4.04 \\
\hline 15 & Arginine & $\mathrm{Y}=5690 \mathrm{X}+430000$ & 0.9998 & $19.88-7952$ & 1.08 & 0.32 & 0.71 & 1.95 & 2.36 & 2.24 & 99.27 & 1.51 & 99.84 & 1.61 & 99.03 & 1.25 \\
\hline 16 & Tyrosine & $\mathrm{Y}=9780 \mathrm{X}+179000$ & 0.9998 & $20.04-2004$ & 2.00 & 0.40 & 2.16 & 3.16 & 1.62 & 2.70 & 99.29 & 2.44 & 99.55 & 2.39 & 101.53 & 2.17 \\
\hline 17 & Uracil & $Y=299 X+35600$ & 0.9990 & $1.97-3936$ & 0.79 & 0.32 & 3.3 & 4.39 & 2.89 & 2.11 & 104.85 & 2.67 & 99.53 & 3.43 & 97.94 & 3.18 \\
\hline 18 & Adenine & $\mathrm{Y}=5810 \mathrm{X}+461000$ & 0.9947 & $1.96-196$ & 1.96 & 0.98 & 1.03 & 2.49 & 3.93 & 3.87 & 104.3 & 1.66 & 101.64 & 4.63 & 98.17 & 2.67 \\
\hline 19 & Hypoxanthine & $Y=2470 X+59300$ & 0.9993 & 19.84-1984 & 9.92 & 3.97 & 3.34 & 4.08 & 3.71 & 1.71 & 99.67 & 1.59 & 99.93 & 1.02 & 99.99 & 1.05 \\
\hline 20 & Guanine & $Y=3780 X+98000$ & 0.9992 & $14.76-1476$ & 5.90 & 2.36 & 1.05 & 3.17 & 3.65 & 3.71 & 103.61 & 1.61 & 101.93 & 2.16 & 101.35 & 2.14 \\
\hline 21 & Dideoxycytidine & $\mathrm{Y}=19900 \mathrm{X}+46500$ & 0.9999 & $4.97-497$ & 1.99 & 0.50 & 2.01 & 3.44 & 2.81 & 2.12 & 98.96 & 2.77 & 101.58 & 4.83 & 98.45 & 2.01 \\
\hline 22 & Thymidine & $Y=2940 X+3200$ & 0.9994 & $2.00-200$ & 1.00 & 0.50 & 1.92 & 3.12 & 3.13 & 3.34 & 100.21 & 3.99 & 103.16 & 3.58 & 95.98 & 1.72 \\
\hline 23 & Cytidine & $\mathrm{Y}=17600 \mathrm{X}+361000$ & 0.9999 & $15.15-3030$ & 1.52 & 0.61 & 2.98 & 3.61 & 3.44 & 2.43 & 100.04 & 3.26 & 99.82 & 2.82 & 99.49 & 2.04 \\
\hline 24 & Uridine & $Y=1260 X+28000$ & 0.9992 & $30.48-3048$ & 15.24 & 3.05 & 2.18 & 3.32 & 3.34 & 3.99 & 97.69 & 2.53 & 100.95 & 2.14 & 99.81 & 2.95 \\
\hline 25 & Dideoxyguanosine & $\mathrm{Y}=13000 \mathrm{X}+143000$ & 0.9997 & $4.95-990$ & 0.99 & 0.25 & 1.94 & 4.28 & 3.52 & 2.67 & 103.88 & 2.35 & 103.01 & 1.51 & 101.45 & 4.16 \\
\hline 26 & Adenosine & $\mathrm{Y}=25400 \mathrm{X}+944000$ & 0.9994 & $19.72-1972$ & 0.99 & 0.39 & 2.54 & 3.39 & 2.53 & 2.66 & 100.91 & 1.36 & 99.72 & 1.08 & 99.76 & 1.17 \\
\hline 27 & Inosine & $\mathrm{Y}=16900 \mathrm{X}+127000$ & 0.9994 & $10.16-1016$ & 5.08 & 2.03 & 2.14 & 2.96 & 2.09 & 3.69 & 101.97 & 2.02 & 102.79 & 4.81 & 95.68 & 2.02 \\
\hline 28 & guanosine & $\mathrm{Y}=15600 \mathrm{X}+346000$ & 0.9999 & $14.70-2940$ & 0.74 & 0.29 & 3.25 & 4.18 & 2.86 & 1.20 & 100.14 & 2.05 & 99.25 & 1.49 & 100.56 & 1.66 \\
\hline 29 & Heterophyllin A & $\mathrm{Y}=4190 \mathrm{X}+10700$ & 0.9996 & $1.25-750$ & 0.75 & 0.38 & 2.1 & 3.35 & 2.67 & 3.28 & 101.52 & 3.32 & 97.57 & 3.34 & 99.96 & 1.96 \\
\hline 30 & Heterophyllin B & $\mathrm{Y}=3480 \mathrm{X}-40800$ & 0.9996 & $5.4-5400$ & 0.54 & 0.22 & 3.28 & 4.37 & 2.55 & 2.87 & 100.42 & 3.6 & 101.35 & 1.99 & 102.45 & 1.1 \\
\hline
\end{tabular}


The LODs and LOQs ranged between $0.13 \mathrm{ng} / \mathrm{mL}$ and $31.13 \mathrm{ng} / \mathrm{mL}$ and $0.40 \mathrm{ng} / \mathrm{mL}$ to $93.40 \mathrm{ng} / \mathrm{mL}$, respectively. The intra- and inter-day precision RSD values ranged from $0.61 \%$ to $3.34 \%$ and $1.93 \%$ to $4.39 \%$, respectively. The satisfactory repeatability presented as RSDs were in the range from $1.01 \%$ to $3.93 \%$. The solution stability presented as RSD was less than $3.99 \%$, indicating the sample was stable when stored at room temperature for $24 \mathrm{~h}$. The recoveries varied between $95.68 \%$ and $104.85 \%$, with RSDs less than $4.83 \%$, demonstrating that this method was validated for all kinds of analytes.

\subsection{Quantification of Cyclopeptides, Nucleosides, and Amino Acids}

The validated analytical method was successfully applied to the simultaneous determination of two cyclopeptides, 16 amino acids, and 12 nucleosides in PR preparations containing 15 samples from different harvesting times, different habitats, and dealt with different processing methods. Each sample was determined three times and the results were reported as mean $\pm \mathrm{SD}$. The total contents of each type of compounds (including cyclopeptides, amino acids, and nucleosides) were statistically evaluated by one-way ANOVA analysis. Typical MRM chromatograms are shown in Figure 1 and the quantitative results are presented in Table 3.

By comparing the amounts, it was found that the compounds of PR from different harvesting times, different habitats, and dealt with using different processing methods were quite different. For different harvesting times, it was clearly shown that the total contents of 30 compounds varied from $3951.04 \mu \mathrm{g} / \mathrm{g}$ to $7858.10 \mu \mathrm{g} / \mathrm{g}$, and in the following order: (highest) 2013.8.6 > 2013.6.15 > 2013.9.12 > 2013.7.9 > 2013.7.15 (lowest). The total contents of each type of compounds were also calculated, the levels of the two cyclopeptides, with total contents of $414.47 \mu \mathrm{g} / \mathrm{g}, 16$ amino acids with total contents of $6158.95 \mu \mathrm{g} / \mathrm{g}$, and 12 nucleosides with total contents of $1284.68 \mu \mathrm{g} / \mathrm{g}$ in PR harvested on 6 August 2013 were significantly higher than that in the herbal materials from other harvesting times based on the significant difference test. As for the habitats, the total contents of 30 constituents ranged from $4267.30 \mu \mathrm{g} / \mathrm{g}$ to $6580.65 \mu \mathrm{g} / \mathrm{g}$, and in order: (highest) Jurong, Jiangsu > Zherong 2, Fujian > Xuancheng, Anhui > Shibing, Guizhou > Zhengrong 1, Fujian (lowest). The 16 amino acids with total contents of $5350.18 \mu \mathrm{g} / \mathrm{g}$, and two cyclopeptides with total contents of $328.40 \mu \mathrm{g} / \mathrm{g}$ in PR from Jurong, Jiangsu were higher than that from other habitats, according to significant difference test. The contents of all 30 compounds in PR dealt with different processing methods ranged from $3096.58 \mu \mathrm{g} / \mathrm{g}$ to $4146.63 \mu \mathrm{g} / \mathrm{g}$, in the order: (highest) oven drying $60{ }^{\circ} \mathrm{C}>$ oven drying $50{ }^{\circ} \mathrm{C}>$ sun drying > sun drying-twisting > oven drying $40^{\circ} \mathrm{C}$ (lowest). For different processing methods, the results of total contents of each type of compounds showed that the levels of the two cyclopeptides $(272.00 \mu \mathrm{g} / \mathrm{g}), 16$ amino acids $(2960.37 \mu \mathrm{g} / \mathrm{g})$, and 12 nucleosides $(914.27 \mu \mathrm{g} / \mathrm{g})$ in PR oven dried at $60{ }^{\circ} \mathrm{C}$ were significantly higher than that handled by other processing methods, which were marked with different letters. The results also demonstrated that UFLC-MS/MS was a very powerful technique for the quantitative analysis of multicomponent of herbal medicines in terms of time savings, sensitivity, and accuracy.

\subsection{GRA of the Samples}

To further evaluate the variation of cyclopeptides, amino acids, and nucleosides in the all tested samples, GRA was performed according to the contents of 30 bioactive components. The normalization treatment of raw data, dimension of the differences of comparing sequences and reference sequences, correlation coefficient of the evaluated samples and the main components, and correlation degree and weight value of the evaluation samples are given in Table S1. The grey comprehensive evaluation values (ri') and quality-rankings are listed in Table 4. S1-S5 were collected at different harvesting times, and the quality ranking in PR from different harvesting times is $\mathrm{S} 4>\mathrm{S} 1>\mathrm{S} 2>\mathrm{S} 5>\mathrm{S} 3$, which indicated that the quality of PR harvested on 6 August 2013 was the better than that of PR from other harvesting times. S6-S10 were collected from five different habitats, and the quality ranking of PR from different habitats is $\mathrm{S} 6>\mathrm{S} 8>\mathrm{S} 10>\mathrm{S} 7>\mathrm{S} 9$, and PR cultivated in Jurong, Jiangsu showed the best quality compared to PR from other habitats. 


\begin{tabular}{|c|c|c|c|c|c|c|c|c|}
\hline $\begin{array}{l}\text { Glycine } \\
2.3 \times 1041 \\
0.0\end{array}$ & 45 & 10 & $\begin{array}{r}\text { Alanine } \\
2.4 \times 106 \\
0.0\end{array}$ & 5 & 10 & $\begin{array}{r}\text { Serine } \\
1.00 \times 106 \\
0.00\end{array}$ & $\prod_{5}^{4.42}$ & 10 \\
\hline $\begin{array}{l}\text { Proline } \\
3.9 \times 1061 \\
0.01\end{array}$ & 5 & 10 & $\begin{array}{r}\text { Valine } \\
3.8 \times 106 \\
0.0\end{array}$ & 5 & 10 & $\begin{array}{r}\text { Threonine } \\
6.8 \times 105 \\
0.0\end{array}$ & $\stackrel{1}{5}_{5}^{3.98}$ & 10 \\
\hline $\begin{array}{l}\text { Leucine } \\
\begin{array}{r}3.0 \times 106 \\
0.00 \\
0\end{array}\end{array}$ & 5 & 10 & $\begin{array}{r}\text { Isoleucine } \\
3.0 \times 106 \\
0.00\end{array}$ & 5 & 10 & $\begin{array}{r}\text { Aspartic acid } \\
1.00 \times 106 \\
0.0\end{array}$ & $\frac{4.25}{5}$ & 10 \\
\hline $\begin{array}{r}\text { Ghutamic acid } \\
6.0 \times 106 \\
0.0\end{array}$ & $\begin{array}{r}5.1 \\
5 \\
5\end{array}$ & 10 & $\begin{array}{r}\text { Lysine } \\
5.8 \times 106 \\
0.00 \\
0\end{array}$ & $\frac{5}{5}$ & 10 & $\begin{array}{r}\text { Methionine } \\
7.5 \times 104 \\
0.0\end{array}$ & 5 & 10 \\
\hline $\begin{array}{r}\text { Histidine } \\
7.6 \times 105 \\
0.0 \\
0\end{array}$ & $\frac{\prod_{5}^{5.1}}{11}$ & 10 & $\begin{array}{r}\text { Phenylalanine } \\
2.4 \times 106 \\
0.0\end{array}$ & 5 & 10 & $\begin{array}{r}\text { Arginine } \\
5.9 \times 106 \\
0.0\end{array}$ & 5. & 10 \\
\hline $\begin{array}{l}\text { Tyrosine } \\
5.9 \times 105 \\
0.00\end{array}$ & 5 & 10 & $\begin{array}{r}\text { Uracil } \\
2.0 \times 105 \\
0.0 \\
\\
\end{array}$ & 5 & 10 & $\begin{array}{r}\text { Adenine } \\
4.9 \times 106 \\
0.0 \\
0\end{array}$ & 5 & 10 \\
\hline $\begin{array}{r}\text { Hypoxanthine } \\
8.0 \times 105 \\
0.0\end{array}$ & 5 & 10 & $\begin{array}{r}\text { Guanine } \\
7.5 \times 105 \\
0.0\end{array}$ & 5 & 10 & $\begin{array}{r}\text { Dideoxycytidin } \\
2.0 \times 106 \\
0.0\end{array}$ & 5 & 10 \\
\hline $\begin{array}{r}\text { Thymidine } \\
4.0 \times 104 \\
0.0\end{array}$ & 5 & 10 & $\begin{array}{l}\text { Cytidine } \\
4.0 \times 106 \\
0.00\end{array}$ & 5 & 10 & $\begin{array}{l}\text { Uridine } \\
\begin{array}{r}6.7 \times 105 \\
0.0\end{array}\end{array}$ & 5 & 10 \\
\hline $\begin{array}{r}\text { Dideoxyguanosi } \\
1.2 \times 106 \\
0.0\end{array}$ & 5 & 10 & $\begin{array}{r}\text { Adenosine } \\
6.0 \times 106 \\
0.0\end{array}$ & 5 & 10 & $\begin{array}{r}\text { Inosine } \\
2.3 \times 106 \\
0.0\end{array}$ & 5 & 10 \\
\hline $\begin{array}{l}\text { Guanosine } \\
4.5 \times 106 \\
0.0\end{array}$ & 5 & 10 & $\begin{array}{r}\text { Heterophyllin A } \\
5.0 \times 105 \\
0.0\end{array}$ & 5 & 10 & $\begin{array}{r}\text { Heterophyllin B } \\
1.9 \times 106 \\
0.0\end{array}$ & 5 & 10 \\
\hline
\end{tabular}

Figure 1. Representative extract ions chromatograms (XIC) of multiple-reaction monitoring (MRM) chromatograms of the 30 investigated compounds. 
Table 3. Contents of 30 compounds in PR $(\mu \mathrm{g} / \mathrm{g}$, mean $\pm \mathrm{SD}, n=3)$.

\begin{tabular}{|c|c|c|c|c|c|c|c|c|c|c|c|c|c|c|c|}
\hline \multirow{2}{*}{ Analyte } & \multicolumn{5}{|c|}{ Different Harvesting Times } & \multicolumn{5}{|c|}{ Different Habitats } & \multicolumn{5}{|c|}{ Different Processing Methods } \\
\hline & S1 ${ }^{\mathrm{a}}$ & S2 & S3 & S4 & S5 & S6 & S7 & S8 & S9 & S10 & S11 & S12 & S13 & S14 & S15 \\
\hline $1^{\mathrm{b}}$ & $\begin{array}{c}104.09 \pm \\
5.85\end{array}$ & $\begin{array}{c}77.34 \pm \\
4.21\end{array}$ & $\begin{array}{c}39.23 \pm \\
9.39\end{array}$ & $\begin{array}{c}117.41 \pm \\
7.27\end{array}$ & $\begin{array}{c}64.98 \pm \\
1.63\end{array}$ & $\begin{array}{c}114.33 \pm \\
3.56\end{array}$ & $\begin{array}{c}76.467 \pm \\
4.05\end{array}$ & $\begin{array}{c}67.99 \pm \\
19.17\end{array}$ & $\begin{array}{c}42.87 \pm \\
2.64\end{array}$ & $\begin{array}{c}100.42 \pm \\
6.25\end{array}$ & $\begin{array}{c}36.00 \pm \\
3.41\end{array}$ & $\begin{array}{c}41.267 \pm \\
2.19\end{array}$ & $\begin{array}{c}33.73 \pm \\
5.66\end{array}$ & $\begin{array}{c}45.53 \pm \\
1.33 \\
\end{array}$ & $\begin{array}{c}56.20 \pm \\
1.71\end{array}$ \\
\hline 2 & $\begin{array}{c}1028.67 \pm \\
23.35\end{array}$ & $\begin{array}{c}910.67 \pm \\
12.86\end{array}$ & $\begin{array}{c}824.00 \pm \\
20.88\end{array}$ & $\begin{array}{c}2468.06 \pm \\
81.89\end{array}$ & $\begin{array}{c}1296.67 \pm \\
27.15\end{array}$ & $\begin{array}{c}1872.67 \pm \\
19.73\end{array}$ & $\begin{array}{c}978.00 \pm \\
44.14\end{array}$ & $\begin{array}{c}1250.67 \pm \\
27.30\end{array}$ & $\begin{array}{c}1130.00 \pm \\
6.93\end{array}$ & $\begin{array}{c}1077.33 \pm \\
22.30\end{array}$ & $\begin{array}{c}568.00 \pm \\
7.21\end{array}$ & $\begin{array}{c}531.33 \pm \\
36.02\end{array}$ & $\begin{array}{c}237.33 \pm \\
3.06\end{array}$ & $\begin{array}{c}696.00 \pm \\
5.29\end{array}$ & $\begin{array}{c}634.00 \pm \\
10.58\end{array}$ \\
\hline 3 & $\begin{array}{c}118.40 \pm \\
3.42\end{array}$ & $\begin{array}{c}107.80 \pm \\
11.72\end{array}$ & $\begin{array}{c}129.53 \pm \\
15.60\end{array}$ & $\begin{array}{c}281.33 \pm \\
11.72\end{array}$ & $\begin{array}{c}131.00 \pm \\
14.91\end{array}$ & $\begin{array}{c}211.13 \pm \\
28.46\end{array}$ & $\begin{array}{c}139.67 \pm \\
7.18\end{array}$ & $\begin{array}{c}143.53 \pm \\
6.45\end{array}$ & $\begin{array}{c}87.93 \pm \\
12.07\end{array}$ & $\begin{array}{c}106.93 \pm \\
13.06\end{array}$ & $\begin{array}{c}52.60 \pm \\
1.31\end{array}$ & $\begin{array}{c}40.53 \pm \\
6.35\end{array}$ & $\begin{array}{c}6.10 \pm \\
4.09\end{array}$ & $\begin{array}{c}27.33 \pm \\
2.66\end{array}$ & $\begin{array}{c}21.20 \pm \\
1.59\end{array}$ \\
\hline 4 & $\begin{array}{c}157.67 \pm \\
5.83\end{array}$ & $\begin{array}{c}148.60 \pm \\
3.70\end{array}$ & $\begin{array}{c}109.00 \pm \\
0.87\end{array}$ & $\begin{array}{c}9.29 \pm \\
0.042\end{array}$ & $\begin{array}{c}300.67 \pm \\
6.43\end{array}$ & $\begin{array}{c}443.33 \pm \\
4.16\end{array}$ & $\begin{array}{c}136.53 \pm \\
3.45\end{array}$ & $\begin{array}{c}195.13 \pm \\
5.16\end{array}$ & $\begin{array}{c}114.33 \pm \\
0.76\end{array}$ & $\begin{array}{c}240.67 \pm \\
3.06\end{array}$ & $\begin{array}{c}106.87 \pm \\
2.32\end{array}$ & $\begin{array}{c}80.73 \pm \\
7.92\end{array}$ & $\begin{array}{c}21.67 \pm \\
0.46\end{array}$ & $\begin{array}{c}106.47 \pm \\
1.33\end{array}$ & $\begin{array}{c}218.00 \pm \\
4.00\end{array}$ \\
\hline 5 & $\begin{array}{c}118.93 \pm \\
1.51\end{array}$ & $\begin{array}{c}139.53 \pm \\
2.80\end{array}$ & $\begin{array}{c}84.13 \pm \\
1.81\end{array}$ & $\begin{array}{c}156.55 \pm \\
1.60\end{array}$ & $\begin{array}{c}74.73 \pm \\
0.64\end{array}$ & $\begin{array}{c}114.067 \pm \\
2.37\end{array}$ & $\begin{array}{c}78.2 \pm \\
1.56\end{array}$ & $\begin{array}{c}85.07 \pm \\
2.00\end{array}$ & $\begin{array}{c}75.00 \pm \\
1.64\end{array}$ & $\begin{array}{c}101.00 \pm \\
2.31\end{array}$ & $\begin{array}{c}71.00 \pm \\
1.60\end{array}$ & $\begin{array}{c}75.80 \pm \\
3.94\end{array}$ & $\begin{array}{c}41.67 \pm \\
0.95\end{array}$ & $\begin{array}{c}70.27 \pm \\
2.08\end{array}$ & $\begin{array}{c}74.80 \pm \\
3.29\end{array}$ \\
\hline 6 & $\begin{array}{c}107.33 \pm \\
2.91\end{array}$ & $\begin{array}{c}141.13 \pm \\
4.23\end{array}$ & $\begin{array}{c}124.27 \pm \\
3.06\end{array}$ & $\begin{array}{c}250.00 \pm \\
2.71\end{array}$ & $\begin{array}{c}134.60 \pm \\
1.91\end{array}$ & $\begin{array}{c}206.67 \pm \\
2.31\end{array}$ & $\begin{array}{c}98.60 \pm \\
7.32\end{array}$ & $\begin{array}{c}127.00 \pm \\
2.27\end{array}$ & $\begin{array}{c}132.33 \pm \\
5.25\end{array}$ & $\begin{array}{c}126.47 \pm \\
3.14\end{array}$ & $\begin{array}{c}122.67 \pm \\
2.00\end{array}$ & $\begin{array}{c}125.13 \pm \\
2.27\end{array}$ & $\begin{array}{c}111.87 \pm \\
2.20\end{array}$ & $\begin{array}{c}146.60 \pm \\
2.09\end{array}$ & $\begin{array}{c}169.20 \pm \\
2.31\end{array}$ \\
\hline 7 & $\begin{array}{c}89.93 \pm \\
0.64\end{array}$ & $\begin{array}{c}80.87 \pm \\
1.01\end{array}$ & $\begin{array}{c}39.00 \pm \\
0.20\end{array}$ & $\begin{array}{c}108.4 \pm \\
1.91\end{array}$ & $\begin{array}{c}53.13 \pm \\
0.95\end{array}$ & $\begin{array}{c}83.73 \pm \\
2.20\end{array}$ & $\begin{array}{c}65.93 \pm \\
1.17\end{array}$ & $\begin{array}{c}65.73 \pm \\
0.76\end{array}$ & $\begin{array}{c}53.20 \pm \\
1.04\end{array}$ & $\begin{array}{c}56.33 \pm \\
0.83\end{array}$ & $\begin{array}{c}27.67 \pm \\
1.14\end{array}$ & $\begin{array}{c}31.27 \pm \\
3.72\end{array}$ & $\begin{array}{c}9.48 \pm \\
0.087\end{array}$ & $\begin{array}{c}24.067 \pm \\
0.12\end{array}$ & $\begin{array}{c}31.80 \pm \\
1.91\end{array}$ \\
\hline 8 & $\begin{array}{c}89.40 \pm \\
0.53\end{array}$ & $\begin{array}{c}80.40 \pm \\
0.92\end{array}$ & $\begin{array}{c}38.80 \pm \\
0.20\end{array}$ & $\begin{array}{c}107.67 \pm \\
1.81\end{array}$ & $\begin{array}{c}52.73 \pm \\
0.95\end{array}$ & $\begin{array}{c}83.27 \pm \\
2.14\end{array}$ & $\begin{array}{c}65.53 \pm \\
1.17\end{array}$ & $\begin{array}{c}65.33 \pm \\
0.76\end{array}$ & $\begin{array}{c}52.87 \pm \\
0.99\end{array}$ & $\begin{array}{c}56.00 \pm \\
0.92\end{array}$ & $\begin{array}{c}27.47 \pm \\
1.14\end{array}$ & $\begin{array}{c}31.07 \pm \\
3.72\end{array}$ & $\begin{array}{c}9.39 \pm \\
0.075\end{array}$ & $\begin{array}{c}23.87 \pm \\
0.12\end{array}$ & $\begin{array}{c}31.53 \pm \\
1.90\end{array}$ \\
\hline 9 & $\begin{array}{c}223.33 \pm \\
13.01\end{array}$ & $\begin{array}{c}240.67 \pm \\
14.05\end{array}$ & $\begin{array}{c}210.53 \pm \\
11.25\end{array}$ & $\begin{array}{c}513.33 \pm \\
19.63\end{array}$ & $\begin{array}{c}276.00 \pm \\
4.00\end{array}$ & $\begin{array}{c}395.33 \pm \\
9.02\end{array}$ & $\begin{array}{c}173.73 \pm \\
5.82\end{array}$ & $\begin{array}{c}250.67 \pm \\
16.29\end{array}$ & $\begin{array}{c}160.6 \pm \\
0.60\end{array}$ & $\begin{array}{c}202.00 \pm \\
2.00\end{array}$ & $\begin{array}{c}90.80 \pm \\
6.77\end{array}$ & $\begin{array}{c}80.33 \pm \\
0.42\end{array}$ & $\begin{array}{c}39.67 \pm \\
2.80\end{array}$ & $\begin{array}{l}79.40 \pm \\
2.42\end{array}$ & $\begin{array}{c}58.13 \pm \\
1.81\end{array}$ \\
\hline 10 & $\begin{array}{c}115.07 \pm \\
2.02\end{array}$ & $\begin{array}{c}116.4 \pm \\
6.51\end{array}$ & $\begin{array}{c}94.27 \pm \\
4.02\end{array}$ & $\begin{array}{c}211.2 \pm \\
12.24\end{array}$ & $\begin{array}{c}98.4 \pm \\
2.46\end{array}$ & $\begin{array}{c}154.07 \pm \\
2.84\end{array}$ & $\begin{array}{c}62.67 \pm \\
0.81\end{array}$ & $\begin{array}{c}104.80 \pm \\
4.73\end{array}$ & $\begin{array}{c}82.53 \pm \\
4.31\end{array}$ & $\begin{array}{c}108.20 \pm \\
1.73\end{array}$ & $\begin{array}{c}54.80 \pm \\
1.78\end{array}$ & $\begin{array}{c}50.00 \pm \\
2.42\end{array}$ & $\begin{array}{c}29.93 \pm \\
1.50\end{array}$ & $\begin{array}{c}53.27 \pm \\
0.76\end{array}$ & $\begin{array}{c}50.33 \pm \\
0.42\end{array}$ \\
\hline 11 & $\begin{array}{c}123.20 \pm \\
4.04\end{array}$ & $\begin{array}{c}126.80 \pm \\
4.19\end{array}$ & $\begin{array}{c}98.80 \pm \\
6.45\end{array}$ & $\begin{array}{c}224.00 \pm \\
0.00\end{array}$ & $\begin{array}{c}104.40 \pm \\
3.27\end{array}$ & $\begin{array}{c}169.20 \pm \\
1.91\end{array}$ & $\begin{array}{c}67.60 \pm \\
2.31\end{array}$ & $\begin{array}{c}112.60 \pm \\
2.42\end{array}$ & $\begin{array}{c}86.07 \pm \\
2.48\end{array}$ & $\begin{array}{c}121.80 \pm \\
0.69\end{array}$ & $\begin{array}{c}59.33 \pm \\
3.11 \\
\end{array}$ & $\begin{array}{c}54.40 \pm \\
4.06\end{array}$ & $\begin{array}{c}33.33 \pm \\
1.67\end{array}$ & $\begin{array}{c}59.07 \pm \\
1.30\end{array}$ & $\begin{array}{c}54.80 \pm \\
2.60\end{array}$ \\
\hline 12 & $\begin{array}{c}3.76 \pm \\
0.18 \\
\end{array}$ & $\begin{array}{c}0.61 \pm \\
0.20 \\
\end{array}$ & $\begin{array}{c}1.00 \pm \\
0.080\end{array}$ & $\begin{array}{c}6.01 \pm \\
1.72 \\
\end{array}$ & $\begin{array}{c}4.79 \pm \\
0.012 \\
\end{array}$ & $\begin{array}{c}8.69 \pm \\
0.69 \\
\end{array}$ & $\begin{array}{c}2.33 \pm \\
0.11 \\
\end{array}$ & $\begin{array}{c}5.41 \pm \\
0.59 \\
\end{array}$ & $\begin{array}{c}0.37 \pm \\
0.071 \\
\end{array}$ & $\begin{array}{c}1.32 \pm \\
0.31 \\
\end{array}$ & $\begin{array}{c}0.27 \pm \\
0.11 \\
\end{array}$ & $\begin{array}{c}0.49 \pm \\
0.00 \\
\end{array}$ & $\begin{array}{c}0.29 \pm \\
0.096 \\
\end{array}$ & $\begin{array}{c}0.30 \pm \\
0.12 \\
\end{array}$ & $\begin{array}{c}0.23 \pm \\
0.030 \\
\end{array}$ \\
\hline 13 & $\begin{array}{c}75.13 \pm \\
1.86\end{array}$ & $\begin{array}{c}108.00 \pm \\
2.27\end{array}$ & $\begin{array}{c}85.93 \pm \\
2.14\end{array}$ & $\begin{array}{c}216.67 \pm \\
4.16\end{array}$ & $\begin{array}{c}124.13 \pm \\
4.22\end{array}$ & $\begin{array}{c}177.80 \pm \\
2.51\end{array}$ & $\begin{array}{c}83.80 \pm \\
2.88\end{array}$ & $\begin{array}{c}295.33 \pm \\
7.02\end{array}$ & $\begin{array}{c}189.33 \pm \\
4.00\end{array}$ & $\begin{array}{c}45.73 \pm \\
0.99\end{array}$ & $\begin{array}{c}107.00 \pm \\
1.25\end{array}$ & $\begin{array}{c}103.00 \pm \\
1.60\end{array}$ & $\begin{array}{c}100.53 \pm \\
2.54\end{array}$ & $\begin{array}{c}130.67 \pm \\
2.87\end{array}$ & $\begin{array}{c}110.27 \pm \\
1.67\end{array}$ \\
\hline 14 & $\begin{array}{c}33.80 \pm \\
13.56\end{array}$ & $\begin{array}{c}46.47 \pm \\
0.61\end{array}$ & $\begin{array}{c}27.07 \pm \\
0.50\end{array}$ & $\begin{array}{c}33.53 \pm \\
4.11\end{array}$ & $\begin{array}{c}41.73 \pm \\
0.76\end{array}$ & $\begin{array}{c}55.67 \pm \\
18.25\end{array}$ & $\begin{array}{c}39.53 \pm \\
19.15\end{array}$ & $\begin{array}{c}35.00 \pm \\
2.62\end{array}$ & $\begin{array}{c}43.87 \pm \\
0.23\end{array}$ & $\begin{array}{c}27.53 \pm \\
11.68\end{array}$ & $\begin{array}{c}32.53 \pm \\
0.31\end{array}$ & $\begin{array}{c}38.27 \pm \\
1.29\end{array}$ & $\begin{array}{c}22.93 \pm \\
0.70\end{array}$ & $\begin{array}{c}33.33 \pm \\
0.50\end{array}$ & $\begin{array}{c}57.00 \pm \\
0.72\end{array}$ \\
\hline 15 & $\begin{array}{c}1352.67 \pm \\
22.03\end{array}$ & $\begin{array}{c}1230.67 \pm \\
160.81\end{array}$ & $\begin{array}{c}1288.00 \pm \\
119.31\end{array}$ & $\begin{array}{c}1205.33 \pm \\
1.15\end{array}$ & $\begin{array}{c}1422.67 \pm \\
90.56\end{array}$ & $\begin{array}{c}1140.67 \pm \\
15.28\end{array}$ & $\begin{array}{c}1172.00 \pm \\
36.39\end{array}$ & $\begin{array}{c}1196.00 \pm \\
23.07\end{array}$ & $\begin{array}{c}1179.33 \pm \\
134.23\end{array}$ & $\begin{array}{c}1304.00 \pm \\
55.46\end{array}$ & $\begin{array}{c}1257.33 \pm \\
79.10\end{array}$ & $\begin{array}{c}1285.33 \pm \\
41.05\end{array}$ & $\begin{array}{c}1366.00 \pm \\
58.41\end{array}$ & $\begin{array}{c}1357.33 \pm \\
71.06\end{array}$ & $\begin{array}{c}1319.33 \pm \\
18.04\end{array}$ \\
\hline 16 & $\begin{array}{c}82.37 \pm \\
35.21\end{array}$ & $\begin{array}{c}55.13 \pm \\
25.84\end{array}$ & $\begin{array}{c}27.49 \pm \\
0.66\end{array}$ & $\begin{array}{c}250.17 \pm \\
5.93\end{array}$ & $\begin{array}{c}67.53 \pm \\
3.19\end{array}$ & $\begin{array}{c}119.56 \pm \\
59.61\end{array}$ & $\begin{array}{c}77.45 \pm \\
5.86\end{array}$ & $\begin{array}{c}101.79 \pm \\
1.82\end{array}$ & $\begin{array}{c}39.07 \pm \\
3.89\end{array}$ & $\begin{array}{c}61.25 \pm \\
0.78\end{array}$ & $\begin{array}{c}45.60 \pm \\
25.46\end{array}$ & $\begin{array}{c}82.40 \pm \\
4.53\end{array}$ & $\begin{array}{c}60.87 \pm \\
0.42\end{array}$ & $\begin{array}{c}68.60 \pm \\
0.58\end{array}$ & $\begin{array}{c}73.53 \pm \\
0.76\end{array}$ \\
\hline
\end{tabular}


Table 3. Cont.

\begin{tabular}{|c|c|c|c|c|c|c|c|c|c|c|c|c|c|c|c|}
\hline \multirow{2}{*}{ Analyte } & \multicolumn{5}{|c|}{ Different Harvesting Times } & \multicolumn{5}{|c|}{ Different Habitats } & \multicolumn{5}{|c|}{ Different Processing Methods } \\
\hline & $S 1^{a}$ & S2 & S3 & S4 & S5 & S6 & S7 & S8 & S9 & S10 & S11 & $\mathrm{S} 12$ & S13 & S14 & S15 \\
\hline Total & $\begin{array}{c}3823.75 \pm \\
37.07^{\mathrm{c}}\end{array}$ & $\begin{array}{c}3611.08 \pm \\
185.73^{c}\end{array}$ & $\begin{array}{c}3221.06 \pm \\
84.97^{\mathrm{d}}\end{array}$ & $\begin{array}{c}6158.95 \pm \\
139.62^{\mathrm{a}}\end{array}$ & $\begin{array}{c}4248.17 \pm \\
51.22^{\mathrm{b}}\end{array}$ & $\begin{array}{c}5350.18 \pm \\
33.29^{\mathrm{a}}\end{array}$ & $\begin{array}{c}3318.05 \pm \\
11.07^{\mathrm{d}}\end{array}$ & $\begin{array}{c}4102.06 \pm \\
75.32^{\mathrm{b}}\end{array}$ & $\begin{array}{c}3469.71 \pm \\
125.79 \mathrm{~d}\end{array}$ & $\begin{array}{c}3736.99 \pm \\
40.10^{c}\end{array}$ & $\begin{array}{c}2659.93 \pm \\
93.99^{\mathrm{b}}\end{array}$ & $\begin{array}{c}2651.36 \pm \\
77.58^{\mathrm{b}}\end{array}$ & $\begin{array}{c}2124.80 \pm \\
43.94^{\mathrm{c}}\end{array}$ & $\begin{array}{c}2922.10 \pm \\
73.45^{\mathrm{a}}\end{array}$ & $\begin{array}{c}2960.37 \pm \\
24.80^{\mathrm{a}}\end{array}$ \\
\hline 17 & $\begin{array}{c}66.07 \pm \\
2.95\end{array}$ & $\begin{array}{c}17.5 \pm \\
4.50\end{array}$ & $\begin{array}{c}9.16 \pm \\
5.42\end{array}$ & $\begin{array}{c}11.97 \pm \\
2.43\end{array}$ & $\begin{array}{c}2.18 \pm \\
2.11\end{array}$ & $\begin{array}{c}12.98 \pm \\
9.39\end{array}$ & $\begin{array}{c}8.46 \pm \\
2.51\end{array}$ & $\begin{array}{c}11.44 \pm \\
2.97\end{array}$ & $\begin{array}{c}20.12 \pm \\
27.33\end{array}$ & $\begin{array}{c}42.4 \pm \\
8.46\end{array}$ & $\begin{array}{c}3.42 \pm \\
0.34\end{array}$ & $\begin{array}{c}3.74 \pm \\
1.06\end{array}$ & $\begin{array}{c}2.78 \pm \\
1.30\end{array}$ & $\begin{array}{c}8.70 \pm \\
1.50\end{array}$ & $\begin{array}{c}2.58 \pm \\
1.30\end{array}$ \\
\hline 18 & $\begin{array}{c}10.74 \pm \\
1.22\end{array}$ & $\begin{array}{c}6.76 \pm \\
2.06\end{array}$ & $\begin{array}{c}9.13 \pm \\
0.60\end{array}$ & $\begin{array}{c}15.35 \pm \\
0.32\end{array}$ & $\begin{array}{c}9.25 \pm \\
0.85\end{array}$ & $\begin{array}{c}15.09 \pm \\
0.53\end{array}$ & $\begin{array}{c}6.99 \pm \\
0.84\end{array}$ & $\begin{array}{c}13.60 \pm \\
0.97\end{array}$ & $\begin{array}{c}3.39 \pm \\
0.55\end{array}$ & $\begin{array}{c}10.31 \pm \\
0.66\end{array}$ & $\begin{array}{c}10.90 \pm \\
0.53\end{array}$ & $\begin{array}{c}4.90 \pm \\
2.53\end{array}$ & $\begin{array}{c}3.92 \pm \\
1.98\end{array}$ & $\begin{array}{l}6.63 \pm \\
1.12\end{array}$ & $\begin{array}{c}7.03 \pm \\
2.51\end{array}$ \\
\hline 19 & $\begin{array}{c}213.33 \pm \\
4.62\end{array}$ & $\begin{array}{c}20.95 \pm \\
7.56\end{array}$ & $\begin{array}{c}10.64 \pm \\
0.60\end{array}$ & $\begin{array}{c}26.00 \pm \\
0.69\end{array}$ & $\begin{array}{c}17.36 \pm \\
1.56\end{array}$ & $\begin{array}{c}31.93 \pm \\
15.37\end{array}$ & $\begin{array}{c}13.79 \pm \\
2.61\end{array}$ & $\begin{array}{c}38.29 \pm \\
16.70\end{array}$ & $\begin{array}{c}15.59 \pm \\
6.51\end{array}$ & $\begin{array}{c}24.87 \pm \\
2.83\end{array}$ & $\begin{array}{c}22.87 \pm \\
1.03\end{array}$ & $\begin{array}{c}14.75 \pm \\
6.28\end{array}$ & $\begin{array}{c}16.27 \pm \\
6.76\end{array}$ & $\begin{array}{c}13.09 \pm \\
8.25\end{array}$ & $\begin{array}{c}32.93 \pm \\
0.58\end{array}$ \\
\hline 20 & $\begin{array}{c}49.60 \pm \\
1.59\end{array}$ & $\begin{array}{c}13.29 \pm \\
0.076\end{array}$ & $\begin{array}{c}11.21 \pm \\
0.49\end{array}$ & $\begin{array}{c}50.40 \pm \\
1.56\end{array}$ & $\begin{array}{c}16.61 \pm \\
1.02\end{array}$ & $\begin{array}{c}33.40 \pm \\
0.87\end{array}$ & $\begin{array}{c}17.25 \pm \\
0.56\end{array}$ & $\begin{array}{c}30.47 \pm \\
0.46\end{array}$ & $\begin{array}{c}3.09 \pm \\
0.00\end{array}$ & $\begin{array}{c}22.13 \pm \\
0.12\end{array}$ & $\begin{array}{c}10.95 \pm \\
0.74\end{array}$ & $\begin{array}{c}11.59 \pm \\
0.89\end{array}$ & $\begin{array}{c}15.69 \pm \\
1.15\end{array}$ & $\begin{array}{c}16.61 \pm \\
0.67\end{array}$ & $\begin{array}{c}17.23 \pm \\
0.63\end{array}$ \\
\hline 21 & $\begin{array}{c}3.02 \pm \\
0.072 \\
\end{array}$ & $\begin{array}{c}7.39 \pm \\
0.095\end{array}$ & $\begin{array}{c}6.97 \pm \\
0.46 \\
\end{array}$ & $\begin{array}{c}21.92 \pm \\
0.23\end{array}$ & $\begin{array}{c}7.50 \pm \\
0.17\end{array}$ & $\begin{array}{c}15.48 \pm \\
0.060\end{array}$ & $\begin{array}{c}7.61 \pm \\
0.50 \\
\end{array}$ & $\begin{array}{c}9.31 \pm \\
0.067\end{array}$ & $\begin{array}{c}1.56 \pm \\
0.071\end{array}$ & $\begin{array}{c}7.85 \pm \\
0.77\end{array}$ & $\begin{array}{c}7.47 \pm \\
0.17 \\
\end{array}$ & $\begin{array}{c}8.62 \pm \\
0.63\end{array}$ & $\begin{array}{c}7.99 \pm \\
0.19\end{array}$ & $\begin{array}{c}7.18 \pm \\
0.21 \\
\end{array}$ & $\begin{array}{c}6.77 \pm \\
0.11\end{array}$ \\
\hline 22 & $\begin{array}{c}37.40 \pm \\
10.86\end{array}$ & $\begin{array}{c}15.54 \pm \\
0.52\end{array}$ & $\begin{array}{c}14.23 \pm \\
13.89\end{array}$ & $\begin{array}{c}41.80 \pm \\
13.34\end{array}$ & $\begin{array}{c}15.08 \pm \\
2.48\end{array}$ & $\begin{array}{c}30.33 \pm \\
5.20\end{array}$ & $\begin{array}{c}20.15 \pm \\
1.37\end{array}$ & $\begin{array}{c}23.47 \pm \\
8.68\end{array}$ & $\begin{array}{c}6.14 \pm \\
7.43\end{array}$ & $\begin{array}{c}20.80 \pm \\
2.31\end{array}$ & $\begin{array}{c}16.25 \pm \\
1.40\end{array}$ & $\begin{array}{c}19.67 \pm \\
0.85\end{array}$ & $\begin{array}{c}19.63 \pm \\
1.61\end{array}$ & $\begin{array}{c}16.64 \pm \\
0.98\end{array}$ & $\begin{array}{c}14.84 \pm \\
0.26\end{array}$ \\
\hline 23 & $\begin{array}{c}64.27 \pm \\
2.00\end{array}$ & $\begin{array}{c}68.27 \pm \\
1.75 \\
\end{array}$ & $\begin{array}{c}57.18 \pm \\
1.70 \\
\end{array}$ & $\begin{array}{c}193.90 \pm \\
1.10\end{array}$ & $\begin{array}{c}68.20 \pm \\
74.01 \\
\end{array}$ & $\begin{array}{c}134.39 \pm \\
1.57\end{array}$ & $\begin{array}{c}130.13 \pm \\
4.32\end{array}$ & $\begin{array}{c}200.73 \pm \\
4.61\end{array}$ & $\begin{array}{c}60.60 \pm \\
0.72 \\
\end{array}$ & $\begin{array}{c}112.40 \pm \\
1.51\end{array}$ & $\begin{array}{c}148.27 \pm \\
3.01\end{array}$ & $\begin{array}{c}128.07 \pm \\
3.92\end{array}$ & $\begin{array}{c}123.60 \pm \\
1.04\end{array}$ & $\begin{array}{c}139.20 \pm \\
2.84\end{array}$ & $\begin{array}{c}156.33 \pm \\
2.83\end{array}$ \\
\hline 24 & $\begin{array}{c}262.00 \pm \\
6.93\end{array}$ & $\begin{array}{c}160.73 \pm \\
8.27\end{array}$ & $\begin{array}{c}98.47 \pm \\
0.99\end{array}$ & $\begin{array}{c}234.00 \pm \\
3.46\end{array}$ & $\begin{array}{c}104.07 \pm \\
3.35\end{array}$ & $\begin{array}{c}174.87 \pm \\
4.24\end{array}$ & $\begin{array}{c}162.93 \pm \\
10.00\end{array}$ & $\begin{array}{c}218.67 \pm \\
4.62\end{array}$ & $\begin{array}{c}155.93 \pm \\
6.33\end{array}$ & $\begin{array}{c}155.07 \pm \\
5.52\end{array}$ & $\begin{array}{c}176.87 \pm \\
2.42\end{array}$ & $\begin{array}{c}167.20 \pm \\
5.33\end{array}$ & $\begin{array}{c}163.00 \pm \\
7.41\end{array}$ & $\begin{array}{c}176.20 \pm \\
2.11\end{array}$ & $\begin{array}{c}193.07 \pm \\
4.00\end{array}$ \\
\hline 25 & $\begin{array}{c}31.20 \pm \\
1.06\end{array}$ & $\begin{array}{c}15.71 \pm \\
0.47\end{array}$ & $\begin{array}{c}14.13 \pm \\
0.58\end{array}$ & $\begin{array}{c}44.33 \pm \\
1.10\end{array}$ & $\begin{array}{c}12.53 \pm \\
0.12 \\
\end{array}$ & $\begin{array}{c}26.67 \pm \\
0.61\end{array}$ & $\begin{array}{c}18.11 \pm \\
0.75\end{array}$ & $\begin{array}{c}23.13 \pm \\
0.31\end{array}$ & $\begin{array}{c}3.34 \pm \\
0.035 \\
\end{array}$ & $\begin{array}{c}15.94 \pm \\
0.20\end{array}$ & $\begin{array}{c}12.85 \pm \\
0.14\end{array}$ & $\begin{array}{c}16.10 \pm \\
0.53\end{array}$ & $\begin{array}{c}16.01 \pm \\
0.33\end{array}$ & $\begin{array}{c}14.67 \pm \\
0.10\end{array}$ & $\begin{array}{c}13.57 \pm \\
0.24\end{array}$ \\
\hline 26 & $\begin{array}{c}141.87 \pm \\
2.19\end{array}$ & $\begin{array}{c}162.47 \pm \\
2.20\end{array}$ & $\begin{array}{c}108.00 \pm \\
5.64\end{array}$ & $\begin{array}{c}259.33 \pm \\
7.57 \\
\end{array}$ & $\begin{array}{c}108.13 \pm \\
2.72 \\
\end{array}$ & $\begin{array}{c}163.60 \pm \\
1.44 \\
\end{array}$ & $\begin{array}{c}182.47 \pm \\
3.97 \\
\end{array}$ & $\begin{array}{c}241.33 \pm \\
3.06\end{array}$ & $\begin{array}{c}146.40 \pm \\
5.05\end{array}$ & $\begin{array}{c}157.53 \pm \\
2.34 \\
\end{array}$ & $\begin{array}{c}168.33 \pm \\
4.10\end{array}$ & $\begin{array}{c}171.53 \pm \\
7.17 \\
\end{array}$ & $\begin{array}{c}159.87 \pm \\
2.60\end{array}$ & $\begin{array}{c}166.20 \pm \\
7.22 \\
\end{array}$ & $\begin{array}{c}176.20 \pm \\
3.47\end{array}$ \\
\hline 27 & $\begin{array}{c}34.27 \pm \\
0.61\end{array}$ & $\begin{array}{c}10.71 \pm \\
0.25\end{array}$ & $\begin{array}{c}6.55 \pm \\
0.012 \\
\end{array}$ & $\begin{array}{c}19.01 \pm \\
0.32\end{array}$ & $\begin{array}{c}6.38 \pm \\
0.19\end{array}$ & $\begin{array}{c}10.67 \pm \\
0.076\end{array}$ & $\begin{array}{c}12.42 \pm \\
0.49\end{array}$ & $\begin{array}{c}17.77 \pm \\
0.095\end{array}$ & $\begin{array}{c}9.55 \pm \\
0.042 \\
\end{array}$ & $\begin{array}{c}10.29 \pm \\
0.061\end{array}$ & $\begin{array}{c}11.31 \pm \\
0.44\end{array}$ & $\begin{array}{c}11.50 \pm \\
0.41\end{array}$ & $\begin{array}{c}10.65 \pm \\
0.33\end{array}$ & $\begin{array}{c}10.97 \pm \\
0.14\end{array}$ & $\begin{array}{c}11.71 \pm \\
0.34\end{array}$ \\
\hline 28 & $\begin{array}{c}228.00 \pm \\
9.17\end{array}$ & $\begin{array}{c}199.47 \pm \\
4.24\end{array}$ & $\begin{array}{c}136.87 \pm \\
4.71 \\
\end{array}$ & $\begin{array}{c}366.67 \pm \\
13.32 \\
\end{array}$ & $\begin{array}{c}139.73 \pm \\
1.79 \\
\end{array}$ & $\begin{array}{c}252.67 \pm \\
1.15 \\
\end{array}$ & $\begin{array}{c}253.33 \pm \\
12.22 \\
\end{array}$ & $\begin{array}{c}349.33 \pm \\
11.02 \\
\end{array}$ & $\begin{array}{c}130.80 \pm \\
0.60\end{array}$ & $\begin{array}{c}193.87 \pm \\
2.12 \\
\end{array}$ & $\begin{array}{c}282.00 \pm \\
3.46\end{array}$ & $\begin{array}{c}247.33 \pm \\
7.02 \\
\end{array}$ & $\begin{array}{c}236.00 \pm \\
2.00\end{array}$ & $\begin{array}{c}251.33 \pm \\
5.03\end{array}$ & $\begin{array}{c}282.00 \pm \\
2.00\end{array}$ \\
\hline Total & $\begin{array}{c}1141.76 \pm \\
11.82^{\mathrm{b}}\end{array}$ & $\begin{array}{c}698.87^{ \pm} \\
22.87^{\mathrm{c}}\end{array}$ & $\begin{array}{c}482.54 \pm \\
14.14^{\mathrm{d}}\end{array}$ & $\begin{array}{c}1284.68 \text { 士 } \\
22.44^{\mathrm{a}}\end{array}$ & $\begin{array}{c}507.02 \pm \\
2.10^{\mathrm{d}}\end{array}$ & $\begin{array}{c}902.07 \pm \\
13.08^{\mathrm{b}}\end{array}$ & $\begin{array}{r}833.63 \pm \\
30.40^{\mathrm{c}}\end{array}$ & $\begin{array}{c}1177.45 \pm \\
19.17^{\mathrm{a}} \\
\end{array}$ & $\begin{array}{r}556.51 \pm \\
29.15^{\mathrm{d}}\end{array}$ & $\begin{array}{c}773.46 \pm \\
15.4^{\mathrm{c}}\end{array}$ & $\begin{array}{c}871.48 \pm \\
9.644^{\mathrm{b}}\end{array}$ & $\begin{array}{l}805.00 \pm \\
25.59^{\mathrm{c}, \mathrm{d}}\end{array}$ & $\begin{array}{c}775.40 \pm \\
15.88^{\mathrm{d}}\end{array}$ & $\begin{array}{c}827.41 \pm \\
5.77^{c}\end{array}$ & $\begin{array}{c}914.27 \pm \\
4.98^{\mathrm{a}}\end{array}$ \\
\hline 29 & $\begin{array}{c}19.20 \pm \\
0.71\end{array}$ & $\begin{array}{c}20.10 \pm \\
0.97\end{array}$ & $\begin{array}{c}13.43 \pm \\
0.50\end{array}$ & $\begin{array}{c}26.47 \pm \\
0.58\end{array}$ & $\begin{array}{c}12.21 \pm \\
0.29\end{array}$ & $\begin{array}{c}19.07 \pm \\
0.70\end{array}$ & $\begin{array}{c}104.07 \pm \\
13.40\end{array}$ & $\begin{array}{c}59.20 \pm \\
3.30\end{array}$ & $\begin{array}{c}26.60 \pm \\
0.80\end{array}$ & $\begin{array}{c}56.00 \pm \\
1.78\end{array}$ & $\begin{array}{c}20.87 \pm \\
0.81\end{array}$ & $\begin{array}{c}18.48 \pm \\
1.08\end{array}$ & $\begin{array}{c}18.38 \pm \\
0.92\end{array}$ & $\begin{array}{c}22.40 \pm \\
1.22\end{array}$ & $\begin{array}{c}24.67 \pm \\
1.01\end{array}$ \\
\hline 30 & $\begin{array}{c}274.00 \pm \\
5.29\end{array}$ & $\begin{array}{c}296.00 \pm \\
15.62\end{array}$ & $\begin{array}{c}234.00 \pm \\
6.93\end{array}$ & $\begin{array}{c}388.00 \pm \\
10.58\end{array}$ & $\begin{array}{c}220.00 \pm \\
0.00\end{array}$ & $\begin{array}{c}309.33 \pm \\
10.26\end{array}$ & $\begin{array}{c}11.55 \pm \\
0.84\end{array}$ & $\begin{array}{c}4.98 \pm \\
0.11 \\
\end{array}$ & $\begin{array}{c}217.33 \pm \\
5.77\end{array}$ & $\begin{array}{c}102.67 \pm \\
7.56\end{array}$ & $\begin{array}{c}198.73 \pm \\
8.25\end{array}$ & $\begin{array}{c}174.07 \pm \\
10.02 \\
\end{array}$ & $\begin{array}{c}178.00 \pm \\
1.74\end{array}$ & $\begin{array}{c}220.00 \pm \\
2.00\end{array}$ & $\begin{array}{c}247.33 \pm \\
3.06\end{array}$ \\
\hline Total & $\begin{array}{c}293.20 \pm \\
5.92^{\mathrm{b}}\end{array}$ & $\begin{array}{c}316.10 \pm \\
16.58^{\mathrm{b}}\end{array}$ & $\begin{array}{c}247.43^{ \pm} \\
6.53^{c}\end{array}$ & $\begin{array}{c}414.47{ }^{ \pm} \\
10.41^{\mathrm{a}}\end{array}$ & $\begin{array}{c}232.21 \pm \\
0.29^{c}\end{array}$ & $\begin{array}{c}328.40 \pm \\
9.81^{\mathrm{a}}\end{array}$ & $\begin{array}{c}115.61 \pm \\
14.15 \mathrm{~d}\end{array}$ & $\begin{array}{c}64.18 \pm \\
3.29^{\mathrm{e}}\end{array}$ & $\begin{array}{c}243.93 \mathrm{~A} \\
5.83^{\mathrm{b}}\end{array}$ & $\begin{array}{c}158.67 \pm \\
7.14^{\mathrm{c}}\end{array}$ & $\begin{array}{c}219.60 \pm \\
8.99^{\mathrm{c}}\end{array}$ & $\begin{array}{c}192.55 \pm \\
10.88^{\mathrm{d}}\end{array}$ & $\begin{array}{c}196.38 \pm \\
0.90^{\mathrm{d}}\end{array}$ & $\begin{array}{c}242.40 \pm \\
1.04^{\mathrm{b}}\end{array}$ & $\begin{array}{c}272.00 \pm \\
4.06^{\mathrm{a}}\end{array}$ \\
\hline
\end{tabular}

${ }^{a}$ The sample No. is same as in Table $5 ;^{\mathrm{b}}$ The analyte No. is the same as in Table $2 ;^{\mathrm{c}}$ Values followed by the same letter in the same row are not significantly different $(p<0.05)$. 
Table 4. Quality sequencing of the samples.

\begin{tabular}{ccc}
\hline Sample & Grey Comprehensive Evaluation Value $\left(\boldsymbol{r}{ }^{\prime}\right)$ & Quality-Ranking \\
\hline S1 & 0.0260 & 2 \\
S2 & 0.0231 & 3 \\
S3 & 0.0211 & 5 \\
S4 & 0.0311 & 1 \\
S5 & 0.0227 & 4 \\
S6 & 0.0300 & 1 \\
S7 & 0.0222 & 4 \\
S8 & 0.0262 & 2 \\
S9 & 0.0201 & 5 \\
S10 & 0.0228 & 3 \\
S11 & 0.0276 & 4 \\
S12 & 0.0278 & 3 \\
S13 & 0.0237 & 5 \\
S14 & 0.0281 & 2 \\
S15 & 0.0298 & 1 \\
\hline
\end{tabular}

Table 5. Summary of information of samples. S1-S5 were collected at different harvesting times. S6-S10 were collected from five different habitats. S11-S15 were dealt with by five different processing methods.

\begin{tabular}{cccc}
\hline Sample No. & Habitats & Harvesting Time & Processing Method \\
\hline S1 & Jurong, Jiangsu & 15 June 2013 & sun drying \\
S2 & Jurong, Jiangsu & 9 July 2013 & sun drying \\
S3 & Jurong, Jiangsu & 15 July 2013 & sun drying \\
S4 & Jurong, Jiangsu & 6 August 2013 & sun drying \\
S5 & Jurong, Jiangsu & 12 September 2013 & sun drying \\
S6 & Jurong, Jiangsu & 10 August 2013 & sun drying \\
S7 & Zherong 1, Fujian & 10 August 2013 & sun drying \\
S8 & Zherong 2, Fujian & 10 August 2013 & sun drying \\
S9 & Shibing, Guizhou & 10 August 2013 & sun drying \\
S10 & Xuancheng, Anhui & 10 August 2013 & sun drying \\
S11 & Jurong, Jiangsu & 10 August 2013 & sun drying \\
S12 & Jurong, Jiangsu & 10 August 2013 & sun drying-twisting \\
S13 & Jurong, Jiangsu & 10 August 2013 & oven drying $40{ }^{\circ} \mathrm{C}$ \\
S14 & Jurong, Jiangsu & 10 August 2013 & oven drying $50{ }^{\circ} \mathrm{C}$ \\
S15 & Jurong, Jiangsu & 10 August 2013 & oven drying $60{ }^{\circ} \mathrm{C}$ \\
\hline
\end{tabular}

S11-S15 were treated with five different processing methods, and the quality ranking of PR handled with different processing methods is S15 > S14 > S12 > S11 > S13, which showed that the quality of PR oven dried at $60{ }^{\circ} \mathrm{C}$ was better than that of PR handled by other processing methods. The present method is suitable for the routine analysis and can contribute to quality control of PR from different harvesting times, different habitats, and dealt with using different processing methods.

\section{Materials and Methods}

\subsection{Chemicals and Reagents}

The reference compounds of glycine (1), alanine (2), serine (3), proline (4), valine (5), threonine (6), leucine (7), isoleucine (8), aspartic acid (9), glutamic acid (10), lysine (11), methionine (12), histidine (13), phenylalanine (14), arginine (15), tyrosine (16), uracil (17), adenine (18), hypoxanthine (19), guanine (20), dideoxycytidine (21), thymidine (22), cytidine (23), uridine (24), dideoxyguanosine (25), adenosine (26), inosine (27), and guanosine (28) were purchased from Shanghai Yuanye-Biotechnology Co., Ltd. (Shanghai, China). Heterophyllin A (29) and heterophyllin B (30) were kindly provided by Professor Ninghua Tan (Kunming Institute of Botany, Chinese Academy of Science, 
Kunming, China). The structures of the 30 reference compounds are shown in Figure 2. The purity of all compounds by HPLC analysis was greater than $98 \%$. Formic acid of MS grade and acetonitrile were purchased from Merck (Darmstajt, Germany). Ultrapure water was prepared using a Milli-Q water purification system (Millipore, Bedford, MA, USA).

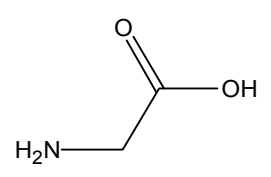

Glycine (1)<smiles>CC(C)C(N)C(=O)O</smiles>

Valine (5)<smiles>NC(CC(=O)O)C(=O)O</smiles>

Aspartic acid (9)

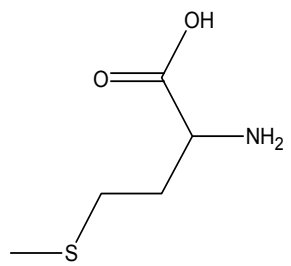

Methionine (12)

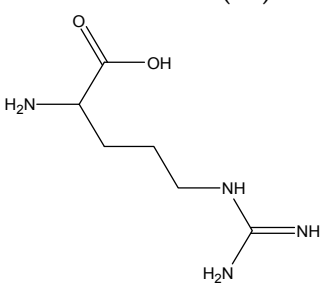

Arginine (15)<smiles>O=C1N=CNC2=NC=NC12</smiles>

Hypoxanthine (19)<smiles>CC(N)C(=O)O</smiles>

Alanine (2)

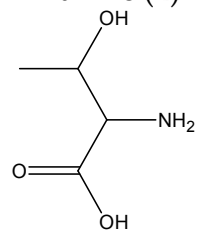

Threonine (6)

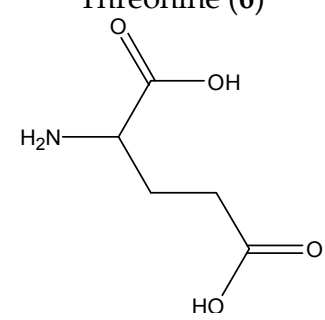

Glutamic acid (10)

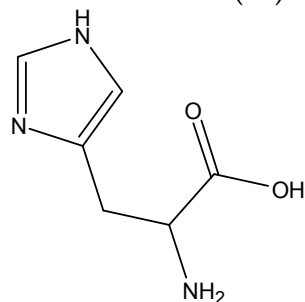

Histidine (13)

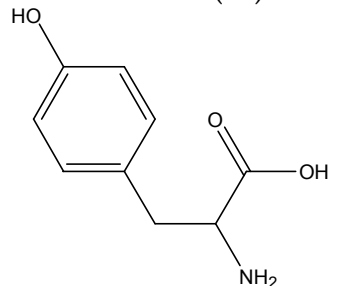

Tyrosine (16)<smiles>Nc1nc2nc[nH]c2c(=O)[nH]1</smiles>

Guanine (20)<smiles>NC(CO)C(=O)O</smiles>

Serine (3)<smiles>CC(C)CC(N)C(=O)O</smiles>

Leucine (7)

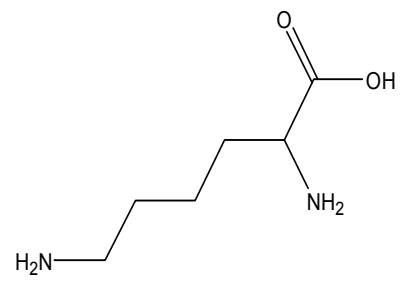

Lysine (11)

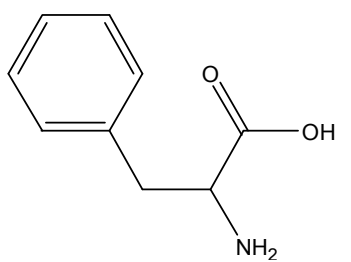

Phenylalanine (14)<smiles>O=c1cc[nH]c(=O)[nH]1</smiles><smiles>Nc1ncnc2[nH]cnc12</smiles>

Uracil (17)<smiles>O=C(NNc1ccn(C2CCC(CO)O2)c(=O)n1)c1ccccc1</smiles>

Dideoxycytidine (21)

Figure 2. Cont 


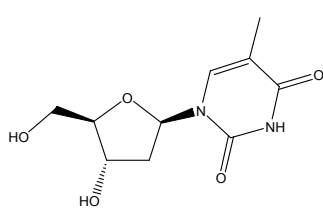

Thymidine (22)<smiles>C[C@]1(CO)CC[C@H](n2cnc3c(=O)[nH]c(N)nc32)O1</smiles>

Dideoxyguanosine (25)<smiles>Nc1nc2c(ncn2[C@@H]2O[C@H](CO)[C@@H](O)[C@H]2O)c(=O)[nH]1</smiles>

Guanosine (28)

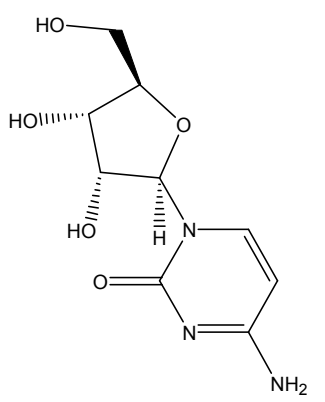

Cytidine (23)<smiles>Nc1ncnc2c1ncn2[C@@H]1O[C@H](CO)[C@@H](O)[C@H]1O</smiles>

Adenosine (26)

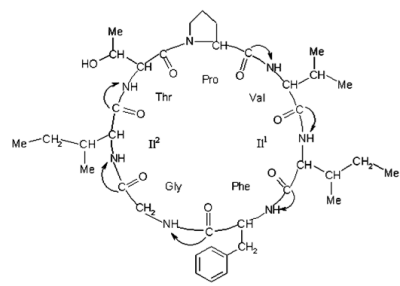

Heterophyllin A (29)

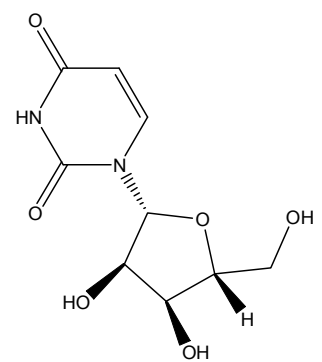

Uridine (24)

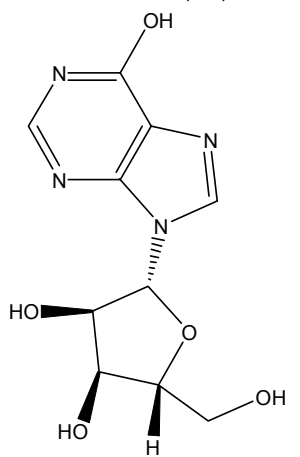

Inosine (27)

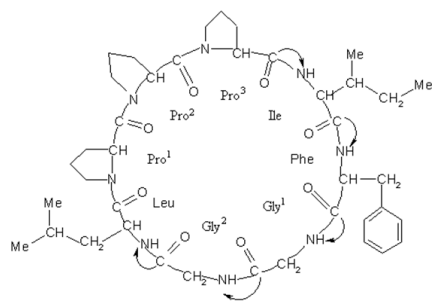

Heterophyllin B (30)

Figure 2. Chemical structures of 30 reference substances.

\subsection{Plant Materials}

Fifteen samples from different harvesting times, different habitats, and dealt with using different processing methods were studied in this research. The samples were authenticated by Prof. Xunhong Liu of the Nanjing University of Chinese Medicine. Five samples (S1-S5) were collected at different harvesting times in June to September 2013 (15 June, 9 July, 15 July, 6 August, 12 September) respectively.

Five samples (S6-S10) were collected from five different habitats (Jurong City, Jiangsu Province, $119^{\circ} 16^{\prime} 49^{\prime \prime}$ N, $31^{\circ} 38^{\prime} 47^{\prime \prime}$ E; Zherong 1 City, Fujian Province, $119^{\circ} 54^{\prime} 2^{\prime \prime}$ N, $27^{\circ} 13^{\prime} 48^{\prime \prime}$ E; Zherong City, Fujian Province, $119^{\circ} 54^{\prime} 2^{\prime \prime}$ N, $27^{\circ} 13^{\prime} 48^{\prime \prime}$ E; Shibing City, Guizhou Province, $108^{\circ} 7^{\prime} 12^{\prime \prime}$ N, $27^{\circ} 1^{\prime} 48^{\prime \prime}$ E; Xuancheng City, Anhui Province, $118^{\circ} 45^{\prime}$ N, $30^{\circ} 56^{\prime} 59^{\prime \prime}$ E) respectively; five samples (S11-S15) were dealt with using different processing methods (sun drying, sun drying-twisting, oven drying $40^{\circ} \mathrm{C}$, oven drying $50^{\circ} \mathrm{C}$, and oven drying $60^{\circ} \mathrm{C}$ ), respectively. Detailed information is shown in Table 5.

\subsection{Preparation of Standard Solution}

A standard stock solution containing 30 reference standards was prepared in water and their concentrations were as follows: 1, $2004 \mathrm{ng} / \mathrm{mL} ; 2,18684 \mathrm{ng} / \mathrm{mL} ; 3,4930 \mathrm{ng} / \mathrm{mL} ; 4,6012 \mathrm{ng} / \mathrm{mL}$; 5, $2012 \mathrm{ng} / \mathrm{mL} ; 6,4064 \mathrm{ng} / \mathrm{mL} ; 7,988 \mathrm{ng} / \mathrm{mL} ; 8,982 \mathrm{ng} / \mathrm{mL} ; 9,5940 \mathrm{ng} / \mathrm{mL} ; 10,4056 \mathrm{ng} / \mathrm{mL}$; 11, $4940 \mathrm{ng} / \mathrm{mL} ; 12,99.6 \mathrm{ng} / \mathrm{mL} ; 13,1976 \mathrm{ng} / \mathrm{mL} ; 14,994 \mathrm{ng} / \mathrm{mL} ; 15,7952 \mathrm{ng} / \mathrm{mL} ; 16,2004 \mathrm{ng} / \mathrm{mL}$; 17, $3936 \mathrm{ng} / \mathrm{mL} ; 18,196 \mathrm{ng} / \mathrm{mL} ; 19,1984 \mathrm{ng} / \mathrm{mL} ; 20,1476 \mathrm{ng} / \mathrm{mL} ; 21,497 \mathrm{ng} / \mathrm{mL} ; 22,200 \mathrm{ng} / \mathrm{mL}$; 23, $3030 \mathrm{ng} / \mathrm{mL} ; 24,3048 \mathrm{ng} / \mathrm{mL} ; 25,990 \mathrm{ng} / \mathrm{mL} ; 26,1972 \mathrm{ng} / \mathrm{mL} ; 27,1016 \mathrm{ng} / \mathrm{mL} ; 28,2940 \mathrm{ng} / \mathrm{mL}$; 
29, $750 \mathrm{ng} / \mathrm{mL} ; 30,5400 \mathrm{ng} / \mathrm{mL}$. This standard stock solution was then diluted with water to a series of appropriate concentrations to generate the calibration curves. The solutions were stored at $4{ }^{\circ} \mathrm{C}$ for a day prior to injection.

\subsection{Preparation of Sample Solutions}

The dried roots were pulverized into homogeneous powders (80 mesh). Powder samples ( $0.1 \mathrm{~g})$ were accurately weighed out and transferred to a $25 \mathrm{~mL}$ conical flask equipped with a stopper, and then water $(20 \mathrm{~mL})$ was added. After accurately weighing the sample and flask, ultrasonication $(250 \mathrm{~W}$, $50 \mathrm{KHz}$ ) was performed at $50{ }^{\circ} \mathrm{C}$ for $60 \mathrm{~min}$ and then water was added to compensate for the weight lost during extraction. After centrifugation $(12,000 \mathrm{rpm}, 10 \mathrm{~min})$ and filtering $(0.22 \mathrm{um}$ membrane filter), the supernatants were stored in a sample plate at $4{ }^{\circ} \mathrm{C}$ before injection into the UFLC system for analysis.

\subsection{Chromatographic and Mass Spectrometric Conditions}

Chromatographic analysis was performed on a Shimadzu SIL-20A XR system (Shimadzu, Kyoto, Japan), consisting of a binary solvent delivery system and an autosampler. Separation was performed on a Waters XBridge Amide $(2.1 \mathrm{~mm} \times 100 \mathrm{~mm}, 3.5 \mu \mathrm{m})$ column. The mobile phase was composed of water with $0.2 \%$ formic acid (A) and acetonitrile with $0.2 \%$ formic acid (B) using a gradient elution of $15 \% \mathrm{~A}$ at $0-2.5 \mathrm{~min}, 15 \%-50 \% \mathrm{~A}$ at $2.5-5 \mathrm{~min}, 50 \% \mathrm{~A}$ at $5-7 \mathrm{~min}, 50 \%-15 \% \mathrm{~A}$ at $7-8 \mathrm{~min}, 15 \% \mathrm{~A}$ at 8-11 min. The flow rate was $0.6 \mathrm{~mL} / \mathrm{min}$, and the column temperature was set at $30^{\circ} \mathrm{C}$.

Mass spectrometry detection was performed using an API5500 triple quadrupole mass (AB SCIEX, Framingham, MA, USA) equipped with an electrospray ionization (ESI) source operating in the negative ion mode. The ESI-MS spectra were acquired in the multiple reaction monitoring (MRM). The parameters in the source were set as follows: GS1 flow $55 \mathrm{~L} / \mathrm{min}$, GS2 flow $55 \mathrm{~L} / \mathrm{min}$, CUR flow $40 \mathrm{~L} / \mathrm{min}$; gas temperature $550^{\circ} \mathrm{C}$; pressure of nebulizer of MS $-4500 \mathrm{~V}$. All MS data were acquired using the Analyst 1.6.2 software to ensure mass accuracy and reproducibility.

\subsection{Validation of the Method}

For the calibration curves, the linearity was verified by plotting the peak areas versus the corresponding concentrations of each analyte. The lowest concentration of working solution for calibration use was diluted with water to a series of appropriate concentrations. The limit of detection (LOD) and limit of quantification (LDQ) of 30 analytes were measured at signal-to-noise values $(\mathrm{S} / \mathrm{N})$ of 3 and 10, respectively. The precision of the developed method was determined by the intra- and inter-day variations. For intra-day test, the mixed standard solutions were analyzed for six replicates with a day, while for inter-day test, the solutions were examined for three consecutive days. The relative standard deviation (RSD) was taken as a measure of precision. To confirm the repeatability, six different analytical sample solutions prepared from the same sample (sample 1) were analyzed and variations were expressed by RSD. To evaluate the stability of the solution, one of the sample solution mentioned above was stored at room temperature and analyzed at $0,2,4,8,12$, and $24 \mathrm{~h}$, respectively. A recovery test was utilized to evaluate the accuracy of this method. A known amount of the 30 standards with low $(80 \%)$, medium $(100 \%)$, and high $(120 \%)$ levels were added into a certain amount of samples $(0.1 \mathrm{~g})$, and then extracted and analyzed with the same procedures. Three replicate extractives at each level were used to calculate the extraction recovery rates for evaluating the method accuracy. The average recovery percentage was calculated by the formula (1):

Recovery $(\%)=($ total amount after spiking - original amount in sample $) /$ spiked amount $\times 100 \%$ 


\subsection{Statistical Analysis}

GRA was carried out to evaluate quality of PR from different harvesting times, different habitats, and dealt with different processing methods, according to the contents of 30 constituents. The data were also statistically evaluated by one-way ANOVA analysis with the aid of SPSS 19.0 software (SPSS Inc., Chicago, IL, USA) to find the significant differences in the contents of different type of 30 compounds. GRA was carried out by the following steps:

\subsubsection{Normalization Treatment of Raw Data}

GRA was carried out to evaluate quality of PR from different harvesting times, different habitats, and dealt with different processing methods according to the contents of 30 components. Assume that there were $n$ samples and each sample had $m$ indexes, the $X_{0}$ was the desired sequence (reference sequence) and the evaluation unit sequence was $X_{i}$. The mean numerical calculation method was used to normalize the raw data:

$$
\mathrm{x}_{\mathrm{i}^{\prime}}^{\prime}(\mathrm{k})=\mathrm{x}_{\mathrm{i}}(\mathrm{k}) / \frac{1}{\mathrm{~m}} \sum_{\mathrm{j}=1}^{\mathrm{m}} \mathrm{x}_{\mathrm{j}}(\mathrm{k})
$$

( $\mathrm{i}=0,1,2,3, \ldots, \mathrm{n} ; \mathrm{k}=0,1,2,3, \ldots, \mathrm{m} ; \mathrm{n}=25, \mathrm{~m}=30$ in this experiment).

\subsubsection{Calculation of the Correlation Coefficient}

The correlation coefficient reflects the accordance between desired sequence and the evaluation unit sequence, the value of correlation coefficient is greater which means that the evaluation unit sequence is closer to desired sequence. The calculation formula of correlation coefficient is:

$$
\xi_{i}(k)=\frac{\min _{i} \min _{k}\left|X_{0}(k)-X_{i}(k)\right|+\rho \max _{i} \max _{k}\left|X_{0}(k)-X_{i}(k)\right|}{\left|X_{0}(k)-X_{i}(k)\right|+\rho \max _{i} \max _{k}\left|X_{0}(k)-X_{i}(k)\right|}
$$

$\rho=0.5, \rho \max _{i} \max _{k}\left|X_{0}(k)-X_{i}(k)\right|$ was the secondary minimum differential value and $\min _{i} \min _{k}\left|X_{0}(k)-X_{i}(k)\right|$ was the secondary maximum differential value.

\subsubsection{Calculation of the Correlation Degree and Weight Value}

It is not convenient to compare the data because there are too much correlation coefficients and the information is dispersive, therefore, it is necessary to concentrate the correlation coefficient to a value and the calculation of mean value is the method of information processing, so that the mean value is the correlation degree. According to the correlation degree $\left(\mathrm{r}_{\mathrm{i}}\right)$, the weight value of evaluated samples can be normalized. The formula of correlation degree is:

$$
\mathrm{r}_{\mathrm{i}}=\frac{1}{\mathrm{~N}} \sum_{\mathrm{k}=1}^{\mathrm{N}} \xi_{\mathrm{i}}(\mathrm{k})
$$

3.7.4. The Grey Comprehensive Evaluation Value

$$
\mathrm{r}_{\mathrm{i}}^{\prime}=\frac{1}{\mathrm{~N}} \sum_{\mathrm{k}=1}^{\mathrm{N}} \omega \mathrm{K} \xi_{\mathrm{i}}(\mathrm{k})
$$

\section{Conclusions}

In this study, an efficient and accurate method was established for the simultaneous quantification of 30 components in PR by using the UFLC-QTRAP-MS/MS technique, which was successfully applied to analyze fifteen PR samples from different harvesting times, different habitats, and dealt with using 
different processing methods. Furthermore, GRA was performed to evaluate the quality of PR samples according to the contents of 30 marker compounds. The results showed that the quality of the different PR samples was obviously different. The quality of PR harvested on 6 August 2013 was the better than PR from other harvesting times. PR cultivated in Jurong, Jiangsu showed the best quality compared to PR from other habitats. The quality of PR dealt with by oven drying $60^{\circ} \mathrm{C}$ was better than PR handled using other processing methods. The proposed method was useful for the overall assessment on the quality of PR, and this study will provide valuable information for revealing the dynamic change law of metabolite accumulation in PR, choosing the suitable harvesting time and reasonable processing method of PR, and exploring the mechanisms responsible for its quality.

Supplementary Materials: Supplementary materials can be accessed at: http://www.mdpi.com/1420-3049/22/ $1 / 13 /$ s1.

Acknowledgments: This work was supported by the National Natural Science Foundation of China (Award No. 81274016; 81473312) and Jiangsu University Advantage Disciplines Construction Engineering Projects (Award ysxk-2014).

Author Contributions: Y.H., S.W., and C.C. carried out the experiments, analyzed data and composed the draft of the manuscript. Y.H., S.W., and Z.L. analyzed data and wrote the manuscript. L.Z. and Q.W. provided the samples of PR. X.L. participated in the supervision of this study and edited the manuscript. H.Z. and Y.Y. proposed the study and helped to perform the experiments. All authors read and approved the final manuscript.

Conflicts of Interest: The authors declare no conflict of interest.

\section{References}

1. Liu, X.H.; Han, L.; Wang, L.J.; Fu, X.S. Study on Quality Standards of Pseudostellaria heterophylla. Chin. Pharm. 2010, 21, 1769-1771.

2. Shen, X.C.; Tao, L.; Bo, S.; Gan, H.R.; Duan, J.A. A meliorated effects of Radix Pseudostellariaeon oxidative stress in rat chronic heart failure induced by acute cardiac infarction. West Chin. J. Pharm. Sci. 2008, 23, 413-416.

3. Fu, X.S.; Liu, X.H.; Xu, H.; Zhou, Y.Z.; Chen, F. Research status and trends of pseudostellariae radix. Chin. J. New Grugs 2012, 21, 757-760.

4. Sheng, R.; Xu, X.; Tang, Q.; Bian, D.; Li, Y.; Qian, C.; He, X.; Gao, X.; Pan, R.; Wang, C.; et al. Polysaccharide of radix pseudostellariae improves chronic fatigue syndrome induced by poly I:C in mice. Evid. Based Complement. Altern. Med. 2011, 2011, 840516. [CrossRef] [PubMed]

5. Fu, X.S.; Liu, X.H.; Xu, H.; Zhou, Y.Z.; Chen, F. Effect of pseudostellaria polysaccharides in diabetic mice by alloxan. Anhui Med. Pharm. J. 2010, 14, 521-522.

6. Pang, W.; Lin, S.; Dai, Q.; Zhang, H.; Hu, J. Antitussive activity of Pseudostellaria heterophylla (Miq.) Pax extracts and improvement in lung function via adjustment of multi-cytokine levels. Molecules 2011, 16, 3360-3370. [CrossRef] [PubMed]

7. Han, C.; Shen, Y.; Chen, J.; Lee, F.S.; Wang, X. HPLC fingerprinting and LC-TOF-MS analysis of the extract of Pseudostellaria heterophylla (Miq.) Pax root. J. Chromatogr. B 2008, 862, 125-131. [CrossRef] [PubMed]

8. Ma, Y.; Hou, Y.; Zou, L.S.; Liu, X.H.; Xu, L.; Yuan, J.D. QTRAP LC-MS/MS analytical study on nucleosides and nucleobases of Pseudostellariae Radix cultivated in different idioplasm resources. J. Chin. Med. Mater. 2015, 38, 711-714.

9. An, K.; He, J.; Wan, Z.M.; Shao, H.W.; Yang, X.C.; Jiao, W. Determination and multivariate statistical analysis of amino acid in Pseudostellaria Heterophylla from different producing areas. Nat. Prod. Res. Dev. 2012, 24, 594-598.

10. Morita, H.; Kayashita, T.; Kobata, H.; Gonda, A.; Takeya, K.; Itokawa, H. Pseudostellarins A-C, new tyrosinase inhibitory cyclic peptides from Pseudostellaria heterophylla. Tetrahedron 1994, 50, 9975-9982. [CrossRef]

11. Han, B.X.; Zhu, Z.X.; Yao, Y.; Li, Y.Y.; Chen, J. A comparative analysis of amino acids and polysaccharides of Pseudostellaria heterophylla from different areas. Chin. Tradit. Pat. Med. 2010, 32, 513-514.

12. Ma, Y.; Hou, Y.; Zou, L.S.; Xu, L.; Liu, X.H.; Lan, C.W.; Luo, Y.Y.; Liu, J.X. Quantitative Determination of Thirteen Nucleosides and Nucleobases in Pseudostellariae Radix in Different Harvest Periods by QTRAP LC-MS/MS. Chin. Pharm. J. 2015, 50, 75-79. 
13. Liu, W.X.; Hu, H.; Liu, X.C.; Duan, Q. Fingerprint of Radix Pseudostellariae by HPLC. Chin. Tradit. Herb. Drugs 2007, 38, 761-764.

14. Liu, X.H.; Wang, M.; Cai, B.C.; Wang, Y.X.; Lin, X.Y. GC-MS Fingerprint of root tuber of Pseudostellaria heterophylla. Chin. Tradit. Herb. Drugs 2007, 38, 113-116.

15. Liu, X.H.; Li, W.; Cai, B.C.; Huang, M.H. Study of capillary electrophoresis fingerprint spectra of Pseudostellariae Radix. J. Nanjing Univ. TCM 2007, 23, 238-240.

16. Zhang, L.L.; Bai, Y.L.; Shu, S.L.; Qian, D.W.; Ou-yang, Z.; Liu, L.; Duan, J.A. Simultaneous quantitation of nucleosides, nucleobases, amino acids, and alkaloids in mulberry leaf by ultra high performance liquid chromatography with triple quadrupole tandem mass spectrometry. J. Sep. Sci. 2014, 37, 1265-1275. [CrossRef] [PubMed]

17. Liu, X.G.; Yang, H.; Cheng, X.L.; Liu, L.; Qin, Y.; Wang, Q.; Qi, L.W.; Li, P. Direct analysis of 18 flavonol glycosides, aglycones and terpene trilactones in Ginkgo biloba tablets by matrix solid phase dispersion coupled with ultra-high performance liquid chromatography tandem triple quadrupole mass spectrometry. J. Pharm. Biomed. Anal. 2014, 97, 123-128. [CrossRef] [PubMed]

18. Zhang, Y.; Guo, L.; Duan, L.; Dong, X.; Zhou, P.; Liu, E.H.; Li, P. Simultaneous determination of 16 phenolic constituents in Spatholobi Caulis by high performance liquid chromatography/electrospray ionization triple quadrupole mass spectrometry. J. Pharm. Biomed. Anal. 2015, 102, 110-118. [CrossRef] [PubMed]

19. Meng, J.; Chen, X.F.; Yang, W.J.; Li, Z.F.; Zhang, Y.; Song, J.H.; Yang, X.W. Evaluation of Bupleuri Radix resources in Qingchuan based on DTOPSIS and grey related degree. Chin. J. Chin. Mater. Med. 2014, 39, 433-437.

20. Liu, W.; Zhang, Y.N.; Pei, J.; Kang, Y.L.; Luo, J.; Chen, C.P.; Ma, Y.T. Grey Relational Analysis of the Relationship between Variety and Quality of Radix Cyathulae. Chin. Pharm. J. 2014, 49, 1796-1801.

Sample Availability: Samples of the compounds 1-30 are available from the authors.

(C) 2016 by the authors; licensee MDPI, Basel, Switzerland. This article is an open access article distributed under the terms and conditions of the Creative Commons Attribution (CC-BY) license (http://creativecommons.org/licenses/by/4.0/). 